\title{
MUFASA: galaxy formation simulations with meshless hydrodynamics
}

\author{
Romeel Davé, ${ }^{1,2,3 \star}$ Robert Thompson ${ }^{4,1}$ and Philip F. Hopkins ${ }^{5}$ \\ ${ }^{1}$ University of the Western Cape, Bellville, Cape Town 7535, South Africa \\ ${ }^{2}$ South African Astronomical Observatories, Observatory, Cape Town 7925, South Africa \\ ${ }^{3}$ African Institute for Mathematical Sciences, Muizenberg, Cape Town 7945, South Africa \\ ${ }^{4}$ National Center for Supercomputing Applications, Champaign-Urbana, IL 61801, USA \\ ${ }^{5}$ California Institute of Technology, Pasadena, CA 91125, USA
}

Accepted 2016 July 23. Received 2016 July 20; in original form 2016 April 5

\begin{abstract}
We present the MUFASA suite of cosmological hydrodynamic simulations, which employs the GIZMO meshless finite mass (MFM) code including $\mathrm{H}_{2}$-based star formation, nine-element chemical evolution, two-phase kinetic outflows following scalings from the Feedback in Realistic Environments zoom simulations, and evolving halo mass-based quenching. Our fiducial $\left(50 h^{-1} \mathrm{Mpc}\right)^{3}$ volume is evolved to $z=0$ with a quarter billion elements. The predicted galaxy stellar mass functions (GSMFs) reproduces observations from $z=4 \rightarrow 0$ to $\lesssim 1.2 \sigma$ in cosmic variance, providing an unprecedented match to this key diagnostic. The cosmic star formation history and stellar mass growth show general agreement with data, with a strong archaeological downsizing trend such that dwarf galaxies form the majority of their stars after $z \sim 1$. We run 25 and $12.5 h^{-1}$ Mpc volumes to $z=2$ with identical feedback prescriptions, the latter resolving all hydrogen-cooling haloes, and the three runs display fair resolution convergence. The specific star formation rates broadly agree with data at $z=0$, but are underpredicted at $z \sim 2$ by a factor of 3, re-emphasizing a longstanding puzzle in galaxy evolution models. We compare runs using MFM and two flavours of smoothed particle hydrodynamics, and show that the GSMF is sensitive to hydrodynamics methodology at the $\sim \times 2$ level, which is sub-dominant to choices for parametrizing feedback.
\end{abstract}

Key words: galaxies: evolution-galaxies: formation.

\section{INTRODUCTION}

Galaxies were first identified over a century ago, yet the physical processes that shape their observable characteristics and evolution remain poorly understood. With the growth of large-scale structure now well specified within the concordance lambda cold dark matter paradigm, the least understood issues surround how the baryonic components behave once the dark matter's gravity is no longer the dominant driver. In particular, much of the uncertainty lies in the nature and physics of feedback processes, i.e. the energy return from processes of star and black hole formation within galaxies that selfregulates their growth. As observations of the galaxy population from the present day back to the epoch of reionization continue to improve, it is an increasingly demanding challenge for models to reproduce such observations using well-motivated and comprehensive input physics, while giving insights into the physical processes responsible for feedback and galaxy assembly.
Successful modern galaxy formation models generally rely on the following basic ingredients (Somerville \& Davé 2015): (i) fuelling of star formation via gravitationally driven gas accretion from the intergalactic medium (IGM); (ii) feedback from massive, young stars that drives large-scale galactic outflows; and (iii) feedback from supermassive black hole accretion that suppresses star formation in massive galaxies. The goal of galaxy formation models is thus to understand and constrain the physics driving these various feedback processes, and particularly how they work in concert to shape galaxies as we observe them via multiwavelength surveys across cosmic time.

Cosmological hydrodynamic simulations have played a major role in the progress towards understanding galaxy formation. The past decade has seen significant advances in both the size and dynamic range of simulations thanks to advancing computing power, as well as the scope and accuracy of the input physics thanks to an improved understanding of the key physical drivers. Such simulations are now able to broadly reproduce primary demographic features of the galaxy population today, along with their evolution to early epochs, to reasonable levels of success (e.g. Davé, Oppenheimer \& Finlator 2011a; Davé, Finlator \& Oppenheimer 
2011b; Genel et al. 2014; Vogelsberger et al. 2014; Schaye et al. 2015; Somerville \& Davé 2015). However, all cosmological-volume models still require 'sub-resolution' recipes for feedback processes, because despite heroic computational efforts it remains infeasible to simultaneously resolve the physics driving feedback at sub-parsec scales within a megaparsec-scale structure formation context. While current models are typically tuned to reproduce key observations such as a galaxy stellar mass function (GSMF) to within a factor of several, they accomplish this using widely differing input physics and numerical implementations, making it difficult to draw robust conclusions regarding the detailed physical processes driving feedback.

One way forward in order to hone in on viable sub-resolution recipes for feedback is to utilize the results of very high resolution simulations of individual galaxies that examine specific feedback processes in detail. This has recently been greatly enabled by the 'zoom' simulation technique in which a particular galaxy and its surroundings are extracted from a larger volume and re-simulated at much higher resolution. In this way, feedback processes are generated more self-consistently and tracked more directly (e.g. Hopkins et al. 2014; Christensen et al. 2016). It is not straightforward to predict population statistics from individual zoom simulations, but their predictions can serve as inputs for the recipes used in cosmologicalscale simulations that model many thousands of galaxies.

A particularly comprehensive set of physical processes was included in the Feedback In Realistic Environments (FIRE) zoom simulations (Hopkins et al. 2014). Using the FIRE simulations, Muratov et al. (2015) derived scaling relations for the mass-loss rate and velocity distribution of outflowing gas, which are key aspects of star formation-driven feedback. Detailed models for self-shielding (e.g. Faucher-Giguere et al. 2010; Rahmati et al. 2013), black hole growth (e.g. Sijacki et al. 2007; Anglés-Alcázar et al. 2015, 2016), and quenching (Gabor et al. 2011) have been investigated to study their emergent trends with a similar strategy in mind. These trends can then be implemented into large-scale, lower-resolution simulations to provide more physically motivated recipes for feedback. Such a multiscale approach offers a promising avenue to assemble robust and well-constrained recipes for feedback processes within cosmological-scale galaxy formation models.

Another important area of progress has been towards increasing the robustness of hydrodynamics solvers. Early simulations were predominantly done using the smoothed particle hydrodynamics (SPH) technique (Hernquist \& Katz 1989; Monaghan 1992). However, this method requires substantial artificial viscosity that compromises the handling of surface instabilities, contact discontinuities, and strong shocks. A reformulation of the SPH equations to explicitly conserve entropy provides some advantages (Springel \& Hernquist 2002; Springel 2005), but still resulted in numerical surface tension that e.g. strongly suppresses Kelvin-Helmholtz instabilities (Agertz et al. 2007). Further reformulations such as SPH-S (Read \& Hayfield 2012) and pressure-SPH (P-SPH; Hopkins 2013) have partially but not fully mitigated these issues.

Adaptive mesh refinement (AMR) galaxy formation codes have also made substantial gains (e.g. The Enzo Collaboration et al. 2014), but their intrinsically Cartesian nature can result in numerical artefacts when applied to the arbitrary 3D geometry and motions associated with galaxy formation. The Voronoi tessellation-based moving mesh code AREPO (Springel 2010) eliminates the Cartesian restrictions, and shows great promise at handling surface instabilities and shocks with arbitrary geometries while retaining Galilean invariance. A downside is that AREPO advects mass across cell boundaries and occasionally must 're-mesh' to prevent highly deformed cells. This necessitates the additional complication of tracer particles in order to track the motion of gas into and out of galaxies (Genel et al. 2014).

Recently, Hopkins (2015) developed a new 'meshless' hydrodynamics method that marries many advantages of SPH and moving mesh approaches. GIZMO is fundamentally a moving mesh-like GODUNOV code, but the 'mesh' is defined by a deformable kernel moving in such a way as to keep the mass within each cell constant. Hence operationally it allows for the advantages of mass tracking and being fully adaptive without re-meshing. Hopkins (2015) showed that GIZMO's 'meshless finite mass' (MFM) approach shows desirable behaviour compared to modern SPH and AMR codes in many relevant idealized test problems. ${ }^{1}$

In this paper we present a new suite of cosmological hydrodynamic simulations of galaxy formation using GIZMO, called the MUFASA simulations. We describe our new recipes for sub-resolution processes (Section 2) based on, wherever possible, the results from zoom simulations or analytic models. These include $\mathrm{H}_{2}$-based star formation, minimal interstellar medium (ISM) pressurization, nonequilibrium cooling and ionization, $\mathrm{SN}$-heated two-phase kinetic winds, evolving halo mass-based quenching, and nine-metal chemical enrichment from Type Ia+II supernovae and stellar evolution. Our highest resolution MUFASA run resolves essentially all hydrogen cooling haloes, while our fiducial $50 \mathrm{~h}^{-1} \mathrm{Mpc}$ volume produces a substantial population of quenched massive galaxies by $z=0$. All are run with exactly the same feedback parameters, and show reasonable resolution convergence. As a basic test of our model, we present predictions for the GSMF across cosmic time, and compare to recent observations and simulations (Section 3). We further compare to observations of the star formation histories, both globally and for individual galaxies (Section 4). We also examine how the choice of hydrodynamics solver and key feedback parameters impact our results (Section 5). Finally, we summarize our findings (Section 6).

\section{CODE DESCRIPTION}

\subsection{GIZMO gravity and hydrodynamics}

We employ a modified version of the gravity plus hydrodynamics solver GIZMO (Hopkins 2015), which is built upon the framework of the GADGET-3 galaxy formation code (Springel 2005). For hydrodynamics, we employ GIZMo's MFM method, which uses a Riemann solver to evolve the fluid in a manner that conserves mass within each fluid element. Each fluid element may then be regarded as a 'particle', though it actually represent a mesh node; we will refer to these as 'gas elements'. Like AREPO, GIZMO offers advantages over $\mathrm{SPH}$ in terms of handling a wide range of fluid problems related to shocks and contact discontinuities, and over AMR codes in terms of avoiding Cartesian artefacts and strictly preserving Galilean invariance.

We employ a cubic spline kernel with 64 neighbours in MFM. Hopkins \& Raives (2016) used 32 neighbours for their detailed magneto-hydrodynamic (MHD) simulations, but we discovered that 64 is better for suppressing occasional instabilities in very diffuse hot gas such as that appearing in the shocked IGM at late epochs. The kernel is used to determine the volume partition between gas elements, thereby defining the effective faces at which the Riemann problem is solved.

\footnotetext{
${ }^{1}$ http://www.tapir.caltech.edu/ phopkins/Site/GIZMO.html
} 
In order to test the sensitivity to hydrodynamics methodology, we will also use the modern P-SPH (Hopkins 2013) and the traditional (entropy-conserving) SPH (T-SPH; Springel \& Hernquist 2002) solvers in GIZMO, with all sub-grid physics options held fixed. SPH requires an artificial viscosity, for which we use the GIZMo default Cullen \& Dehnen (2010) viscosity as described in Hopkins (2015). To isolate the impact of the hydro solver, we use the same cubic spline kernel with 64 neighbours, even though a quintic spline gives better results in some circumstances. ${ }^{2}$

The gravity is evolved using a Tree-Particle-Mesh approach that is based on GADGET-3 (Springel 2005). We use adaptive gravitational softening throughout for all particles (Hopkins 2015), enclosing 64 particles with a minimum (Plummer-equivalent) softening length set to 0.5 per cent of the mean interparticle spacing. For more details on GIZMO, including other choices such as the timestep limiter (Durier \& Dalla Vecchia 2012) and the implementation of adaptive softening, see Hopkins (2015).

\subsection{Radiative cooling and heating}

We use the GRACKLE-2.1 chemistry and cooling library (Kim et al. 2014; The Enzo Collaboration 2014), which includes primordial and metal-line cooling, evolved isochorically on the cooling time-scale in an operator-split way over the system timestep. In other words, we do the non-radiative cooling/heating first over the system timestep, then apply radiative cooling using GRACKLE, where the radiative part is sub-stepped isochorically on the cooling time-scale.

We employ GRACKLE in non-equilibrium mode for primordial elements, thereby tracking e.g. the input of latent heat during reionization. Metal-line cooling is computed using CLOUDY (Ferland 2004) tables self-consistently including the impact of photoionization, in this case assuming ionization equilibrium. A spatially uniform photoionizing background is assumed, for which we employ the determination by Faucher-Giguere et al. (2009), updated in 2011. We do not employ the more recent Haardt \& Madau (2012) determination owing to concerns regarding its low $\mathrm{H}$ I ionization rate at $z=0$ which has spurred the so-called photon underproduction crisis (Kollmeier et al. 2014). In any case, galaxy properties that we consider here are insensitive to reasonable choices of the photoionizing background.

\subsection{Star formation}

To form star particles out of gas, we employ a molecular gasbased star formation prescription following Krumholz, McKee \& Tumlinson (2009, hereafter KMT). A fuller description is available in Krumholz, McKee \& Tumlinson (2011) and Thompson et al. (2014); here we briefly summarize the approach.

KMT provides an approximate solver for $\mathrm{H}_{2}$ formation at the (relatively) coarse resolution available to us in cosmological volumes. With well-motivated approximations, this yields

$$
f_{\mathrm{H} 2}=1-0.75 \frac{s}{1+0.25 s} \text {, }
$$

\footnotetext{
${ }^{2}$ Dehnen \& Aly (2012) found that SPH similar to T-SPH using a cubic spline kernel with 64 neighbours may, in strong shearing flows, be subject to particle pairing instabilities that result in lowering the effective resolution. We thus ran the same T-SPH simulation with a quintic spline with 64 neighbours, and the results were essentially identical. Hence this pairing instability is evidently not impacting our results.
}

where

$s=\frac{\ln \left(1+0.6 \chi+0.01 \chi^{2}\right)}{0.0396 Z\left(\Sigma / \mathrm{M}_{\odot} \mathrm{pc}^{-2}\right)}$,

where $Z$ is the metallicity in solar units, $\chi$ is a function of metallicity given in KMT, and $\Sigma$ is the column density calculated using the Sobolev approximation; see below. We impose a minimum metallicity of $10^{-3} Z_{\odot}$ solely for the purposes of this computation. We further require a minimum density for star formation of $n_{\mathrm{H}} \geq 0.13 \mathrm{~cm}^{-3}$; gas above this density will hereafter be referred to as 'ISM gas'. In most cases, the densities at which $\mathrm{H}_{2}$ dominates is higher than this (e.g. $n_{\mathrm{H}} \sim 0.5-1 \mathrm{~cm}^{-3}$ for solar metallicity) but for individual high-metallicity gas elements the KMT formula can yield $\mathrm{H}_{2}$ formation, and hence star formation, below our ISM density threshold; we explicitly do not allow this.

We make two minor adjustments to the KMT algorithm regarding sub-grid clumping. First, KMT assumed a constant sub-resolution clumping factor in the ISM of $\mathcal{C}=30$. This factor appears in the rate coefficient for forming $\mathrm{H}_{2}$ on dust grains (see section 2.2 of Krumholz et al. 2011). It is clear that a higher resolution simulation should assume less clumping - in the limiting case of infinite resolution, the clumping should be fully resolved and hence $\mathcal{C}$ should be unity. Thus it makes physical sense to reduce $\mathcal{C}$ in higher resolution simulations. In our case, we assume that $\mathcal{C}=30$ in our fiducial simulation with $\epsilon=0.5 h^{-1} \mathrm{kpc}$ (see Section 2.10), where $\epsilon$ is our minimum comoving gravitational softening length, but we further scale $\mathcal{C} \propto \epsilon$ in our other runs (see Table 1). By this scaling, a simulation with $\epsilon \sim 25 \mathrm{pc}$ would have $\mathcal{C}=1$; our cosmological runs here do not reach this limit. The net effect of scaling $\mathcal{C}$ is to make star formation slightly less efficient in higher resolution simulations, leaving them slightly more gas rich.

Our second adjustment involves the calculation of $\Sigma$. KMT assumes that self-shielding against $H_{2}$ dissociating radiation within the ISM is governed by the local column density $\Sigma$ as calculated using the Sobolev approximation, namely $\Sigma=\rho^{2} /|\nabla \rho|$ where $\rho$ is the gas density. We compute these quantities in the usual way from the gas elements, but we must further account for sub-resolution clumping. If the clumping factor $\mathcal{C}$ represents the typical density contrast of the sub-resolution clumps, then the column density increase within these clumps is approximately, by dimensional arguments, $\mathcal{C}^{2 / 3}$. We thus increase $\Sigma$ by a factor of $\mathcal{C}^{2 / 3} \approx 10$ to account for this. ${ }^{3}$

Given an $\mathrm{H}_{2}$ fraction from KMT, the star formation rate (SFR) for a given eligible gas element is given by a Schmidt (1959) law, namely

$\frac{\mathrm{d} M_{*}}{\mathrm{~d} t}=\epsilon_{*} \frac{\rho f_{\mathrm{H} 2}}{t_{\mathrm{dyn}}}$,

where $f_{\mathrm{H} 2}$ is the molecular fraction for a given gas element, and $t_{\text {dyn }}=1 / \sqrt{G \rho}$ is the local dynamical time. We set the efficiency of star formation to be $\epsilon_{*}=0.02$ (Kennicutt 1998).

Star particles are spawned from gas elements stochastically. Each MFM fluid element spawns a single star particle of the same mass. Previous simulations (e.g. Oppenheimer \& Davé 2008) often spawn multiple star particles, reducing the gas mass by a fraction each time.

\footnotetext{
${ }^{3}$ The factor of 10 is appropriate for our fiducial large volume. This factor should vary as $\mathcal{C}$ varies, but owing to an oversight this was not implemented for the higher resolution runs. We subsequently ran test simulations to determine that the results are not significantly different if we scale $\Sigma \propto \mathcal{C}^{2 / 3}$, though it does lower star formation very slightly. Future runs will use this scaling.
} 
Table 1. $2 \times 512^{3}$-particle MUFASA runs, with reduced $\chi_{v}^{2}$ values relative to observed GSMFs at $z=0-6$.

\begin{tabular}{|c|c|c|c|c|c|c|c|c|c|c|c|}
\hline Name & $L$ & $\epsilon$ & $m_{\text {gas }}$ & $m_{\text {dark }}$ & $m_{\mathrm{gal}}$ & $\chi_{z=0}^{2}$ & $\chi_{z=1}^{2}$ & $\chi_{z=2}^{2}$ & $\chi_{z=3}^{2}$ & $\chi_{z=4}^{2}$ & $\chi_{z=6}^{2}$ \\
\hline $\mathrm{m} 50 \mathrm{n} 512$ & 50 & 0.5 & $1.82 \times 10^{7}$ & $9.6 \times 10^{7}$ & $5.8 \times 10^{8}$ & 0.20 & 0.98 & 1.11 & 0.23 & 1.32 & 1.97 \\
\hline $\mathrm{m} 25 \mathrm{n} 512$ & 25 & 0.25 & $2.28 \times 10^{6}$ & $1.2 \times 10^{7}$ & $7.3 \times 10^{7}$ & - & - & 1.04 & 0.08 & 1.24 & 1.00 \\
\hline $\mathrm{m} 12.5 \mathrm{n} 512$ & 12.5 & 0.125 & $2.85 \times 10^{5}$ & $1.5 \times 10^{6}$ & $9.1 \times 10^{6}$ & - & - & 0.19 & 0.02 & 0.38 & 0.26 \\
\hline
\end{tabular}

Note. $L$ is the box size in comoving $h^{-1} \mathrm{Mpc}$.

$\epsilon$ is the minimum gravitational softening length in comoving $h^{-1} \mathrm{kpc}$ (equivalent Plummer).

$m_{\mathrm{gal}}$ is the galaxy stellar mass resolution limit in $M_{\odot}$ (32 star particle masses).

However, large instantaneous changes to an element's gas mass can cause instabilities in MFM, so we avoid this by converting the gas element fully into a collisionless star particle all at once, conserving mass and momentum.

\subsection{Resolving the Jeans mass in the ISM}

As the density increases and the cooling rate becomes large, the Jeans mass can become smaller than the resolved mass in the simulation. If cooling is allowed to proceed at these high densities, the fragmentation that should proceed to small scales (and thus be captured by our star formation law) is instead 'bottlenecked' at large scales, producing artificially coherent clumping (Schaye \& Dalla Vecchia 2008).

To avoid this, we apply an artificial pressure above a density threshold $n_{\text {th }}$ that explicitly suppresses fragmentation below the scale of the smoothing volume, namely:

$n_{\text {th }}=\frac{3}{4 \pi \mu m_{\mathrm{p}}}\left(\frac{5 k_{\mathrm{B}} T_{0}}{G \mu m_{\mathrm{p}}}\right)^{3}\left(\frac{1}{N_{\mathrm{ngb}} m_{\mathrm{g}}}\right)^{2}$,

where $\mu$ is the molecular weight calculated assuming fully neutral gas, $m_{\mathrm{g}}$ is the mass per gas element in our simulation, and $N_{\mathrm{ngb}}=64$ is the number of neighbours.

We assume that the temperature at the density threshold is $T_{0}=10^{4} \mathrm{~K}$. Above this density, we then set the gas element temperature to the Jeans minimum temperature:

$T_{\mathrm{JMT}}=T_{0}\left(n / n_{\mathrm{th}}\right)^{1 / 3}$.

If $n_{\text {th }}$ is below our nominal star formation density threshold, we set it to this value; this occurs in our largest volume. Meanwhile, our smallest volume has $n_{\mathrm{th}}=11.6 \mathrm{~cm}^{-3}$, while our intermediate volume has $n_{\text {th }}=0.18 \mathrm{~cm}^{-3}$.

This prescription is quite similar to the $P \propto T^{4 / 3}$ equation of state imposed by Schaye \& Dalla Vecchia (2008), except that we vary the threshold density with the simulation resolution such that at higher resolution, we do not artificially pressurize the ISM as strongly. Hence our approach automatically takes advantage of high numerical resolution, when present, as much as possible (e.g. Thompson et al. 2016).

\subsection{Feedback from massive stars}

As necessitated by our cosmological resolution, we employ a subresolution approach to modelling feedback from massive stars. The basic approach is to use kinetic feedback (Springel \& Hernquist 2003a; Oppenheimer \& Davé 2006), as this has generally been shown to provide stable and well-converged results for cosmological galaxy formation simulations, and allows more explicit control over the numerical experiments regarding feedback.

In our feedback prescription, we assume that massive stars launch winds that drive material out of galaxies through a combination of
Type II supernovae (SNII), radiation pressure, and stellar winds. We do not track these phenomena in the code owing to a lack of resolution, but rather characterize the net effect using two free parameters, namely the mass loading factor $\eta$ defined as the mass outflow rate relative to the SFR, and the wind speed $v_{\mathrm{w}}$ representing the velocity with which the wind is launched. Both parameters can in principle scale with galaxy properties and redshift. Outflow fluid elements are launched in the direction of $\pm \boldsymbol{v} \times \boldsymbol{a}$, in order to have a broadly collimated outflow perpendicular to the disc, and the sign is chosen such that it is launched in the hemisphere pointing away from the host galaxy. Later we will show that this choice has no discernible impact versus ejecting gas isotropically.

\subsubsection{FIRE wind scalings}

Our general strategy is to directly employ outflow scalings derived from the FIRE simulations (Hopkins et al. 2014), which selfconsistently drive winds from galaxies using supernova (SN) feedback, radiation from massive stars, and stellar winds, and have been successful at reproducing a range of observed galaxy properties in individual galaxy zoom simulations.

Muratov et al. (2015) found in the FIRE simulations that the outflow rate was most tightly correlated with stellar mass, and this scaling was essentially independent of redshift. Their best-fitting relation of the mass outflow rate at $0.25 R_{\mathrm{vir}}$ is given by

$\eta=3.55\left(\frac{M_{*}}{10^{10} \mathrm{M}_{\odot}}\right)^{-0.351}$,

where $M_{*}$ is the galaxy stellar mass. We implement this relation directly into our code. We compute $M_{*}$ using an on-the-fly galaxy finder, which we describe in Section 2.6. To account for the very early evolution of galaxies when little or no stellar mass has yet formed, we place a floor on the stellar mass used in the above equation of eight gas element masses.

For the wind speed, we likewise take the scaling of $v_{\mathrm{w}}$ with galaxy properties from the FIRE simulations. Their best-fitting formula is $v_{\mathrm{w}}=0.854 v_{\mathrm{c}}^{1.12}$, where $v_{\mathrm{c}}$ is the galaxy's circular velocity. We will describe how we obtain $v_{\mathrm{c}}$ from our on-the-fly galaxy finder in Section 2.6. In FIRE, this formula reflects the mean velocities across a shell at $0.25 R_{\mathrm{vir}}$. To account for the fact that our wind launches are typically at a much smaller radius well within the ISM, we add an extra velocity kick $\Delta v_{0.25}$ corresponding to the gravitational potential difference between the wind launch radius and $0.25 R_{\text {vir }}$ (Łokas et al. 2001). We assume a Navarro, Frenk \& White (1997) profile with a concentration $c=9\left(M_{\text {halo }} / 10^{10} \mathrm{M}_{\odot}\right)^{-0.15}$, where we compute the halo mass as the galaxy baryonic mass times $\Omega / \Omega_{\mathrm{b}}$. This typically increases the wind velocity by up to $\sim v_{\mathrm{c}}$ in small galaxies, and by significantly less in large galaxies.

Still, $\Delta v_{0.25}$ only accounts for gravitational slowing, while hydrodynamic slowing is likely to be dominant (Oppenheimer \& Davé 
2008). Hence we further increase the multiplier of the outflow velocity, by around a factor of 2 above the Muratov et al. (2015) value, comparable to their 95 th percentile wind velocity, and also comparable to the outflow velocities measured leaving the disc in the zoom simulations of Christensen et al. (2016). We choose to normalize this multiplier to the FIRE scaling at $200 \mathrm{~km} \mathrm{~s}^{-1}$. Hence the velocity formula used in MUFASA is

$v_{\mathrm{w}}=2\left(\frac{v_{\mathrm{c}}}{200}\right)^{0.12} v_{\mathrm{c}}+\Delta v_{0.25}$.

Finally, we add a random tophat scatter of \pm 10 percent to $v_{\mathrm{w}}$. The resulting wind launch velocities are typically $\sim 2-3 v_{\mathrm{c}}$, which is comparable to the observed maximum outflow speeds in lowredshift (Martin 2005) and high-redshift (Steidel et al. 2010) galaxies.

\subsubsection{Two-phase winds}

Winds are often observed to be flowing out of nearby galaxies (e.g. M82) partly in hot gas, putatively heated by SN explosions and the resulting shocks. Previous kinetic wind models (Davé et al. 2011a; Vogelsberger et al. 2014) have generally not included this form of energy injection, even though their simulations lack the resolution to properly capture SNe shock heating in the ISM. Winds are also seen in cool form, mainly via low-ionization absorption lines. Hence it is clear that there are at least two phases for outflowing gas.

In MUFASA, we model two-phase winds by randomly selecting outflowing gas to be 'cool' or 'hot'. Cool gas is ejected at $T \approx$ $10^{3} \mathrm{~K}$, although they quickly heat to $\sim 10^{4} \mathrm{~K}$ via photo-ionization once they are outside the ISM. For the hot wind, we directly employ a fraction of the SN energy not used for kinetic ejection into thermal heating of the wind. For a Chabrier initial mass function (IMF), each solar mass of stars produces 0.01028 stars with masses above $8 \mathrm{M}_{\odot}$. We assume each of these becomes an $\mathrm{SN}$ which produces $10^{51} \mathrm{erg}$, hence the specific energy production from $\mathrm{SNe}$ is $u_{\mathrm{SN}}=5.165 \times 10^{15} \mathrm{erg} \mathrm{g}^{-1}$.

The kinetic energy used by the wind is $\frac{1}{2} m_{\mathrm{w}} v_{\mathrm{w}}^{2}=\frac{1}{2} \eta m_{\mathrm{g}} v_{\mathrm{w}}^{2}$, where we assume that the total wind mass ejected owing to a gas mass of $m_{\mathrm{g}}$ forming into stars is $\eta m_{\mathrm{g}}$. If each wind fluid element has mass $m_{\mathrm{g}}$, then the specific energy utilized by the wind is $\frac{1}{2} \eta v_{\mathrm{w}}^{2}$.

The specific thermal energy added to each hot wind gas element is then

$\Delta u=u_{\mathrm{SN}}-\frac{1}{2} \eta v_{\mathrm{w}}^{2}$.

If $\Delta u<0$ we add no heat; physically, this can represent a situation where other wind mechanisms such as radiation pressure are primarily responsible for driving winds. We have found that so long as the heating raises the typical temperature above a few times $10^{5} \mathrm{~K}$ to get it past the helium and $\mathrm{O}$ VI cooling peaks (which $\Delta u$ almost always does), then the impact on galaxies is relatively insensitive to the exact amount of heating.

We define a free parameter $f_{\text {hot }}$ that determines the fraction of the wind that is ejected hot. Each wind element is then randomly chosen to be either hot or cool, based on the probability $f_{\text {hot }}$. In MUFASA, we choose $f_{\text {hot }}=0.3$, motivated by ISM-scale simulations of SN-driven winds that suggest roughly 30 per cent of the material is ejected in hot form (T. Naab, private communication). Hence our two-phase wind is assumed to be majority cool, but containing a substantial component of hot gas.

\subsubsection{Hydrodynamic decoupling and recoupling}

No hydrodynamics code is able to properly evolve a single fluid element (cell or particle) moving with a high Mach number $\mathcal{M}$ through its surroundings. This is simply a limitation of resolution, and is not mitigated by the use of an improved hydrodynamics solver in MFM: a single fluid element will never be able to capture the subelement mixing and dissipation with its surrounding gas. For this reason, it is necessary to make some approximation regarding the interactions of the outflowing gas when passing through the ISM. Ideally, one would like to follow the detailed evolution of gas mixing with its surroundings via surface instabilities, but this is non-trivial, since the Kelvin-Helmholtz mixing time is generally very short. Instead, we follow early works by Springel \& Hernquist (2003a) and Oppenheimer \& Davé (2006, 2008), along with recent simulations such as Illustris (Vogelsberger et al. 2014), and employ decoupled winds. That is, we turn off hydrodynamic forces on outflowing gas until such time as it 'recouples' back to its surrounding gas. The physical motivation is that winds are often seen to blow channels through the ISM that likely allow the relatively unfettered escape of a coherent flow. Moreover, this makes physical sense since our outflow scalings are taken from FIRE simulations who measured their wind properties well outside the ISM.

The criteria for recoupling has historically been chosen via a density threshold, e.g. 10 per cent of the ISM density threshold, and a time limit, e.g. enough to free-stream for tens of kpc (e.g. Springel \& Hernquist 2003a). Here, we add a new recoupling criterion: That the velocity of the outflowing gas element be similar to its surroundings. This criterion additionally allows recoupling when the gas has a velocity that can be properly handled by the hydrodynamics solver.

Specifically, our recoupling criterion requires that the wind gas element has a velocity difference with respect to surrounding gas of less than 50 per cent of the local sound speed $(\mathcal{M}<0.5)$. The sound speed is calculated from the kernel-weighted temperature of surrounding gas at the wind element's position, and the velocity difference is summed over all neighbours with kernel weighting. We exclude all other decoupled wind gas when computing quantities for the surrounding gas. Using 20 per cent or 100 per cent rather than 50 per cent did not yield appreciably different results. We further turn off radiative cooling while the wind is decoupled, in order to allow hot gas in the wind to deposit their thermal energy into the circumgalactic medium (CGM).

Gas elements satisfying the Mach number criterion are recoupled regardless of surrounding density or time, so long as they have a density that is less than the ISM density. But there are cases where this recoupling criterion is not satisfied for a long while or until far away, particularly in low-mass haloes where the outflows are typically ejected into very low density gas. Hence we also employ a density limit of 1 per cent of the ISM threshold density, and a time limit of 2 per cent of the Hubble time at launch which is comparable to a disc dynamical time; reaching either of these criteria automatically triggers recoupling as well. In the end, the total fraction of decoupled gas is always well below 1 per cent, reaching a maximum of around $0.2-0.3$ per cent at $z \sim 2$.

\subsection{Fast, approximate friends-of-friends}

Equation (6) requires $M_{*}$ to determine $\eta$, and equation (7) requires $v_{\mathrm{c}}$ to determine the outflow velocity. To determine $M_{*}$ and $v_{\mathrm{c}}$, we employ a fast approximate friends-of-friends (FOF) galaxy finder on the fly during the simulation run. This FOF finder groups stars and ISM gas into baryonic galaxies. It is approximate because it 
finds neighbours within a cube of side $2 \mathcal{L}$, where $\mathcal{L}$ is the linking length, rather than a sphere of radius $\mathcal{L}$. This greatly improves computation speed while having minimal impact on the identified galaxies, thus enabling the code to run this FOF finder at essentially every timestep. We calibrate $\mathcal{L}=0.0056$ times the mean interparticle spacing in order to obtain the same mass function as that obtained by using the Spline Kernel Interpolative Denmax (SKID; e.g. Kereš et al. 2005) galaxy finder. Our results are insensitive to variations in $\mathcal{L}$ by a factor of a few around our chosen value, but we choose this value since it is the minimum hydrodynamic kernel size, which is 40 per cent of the minimum softening length, multiplied by 2.8 which is the conversion from Plummer-equivalent softening to the actual smoothing kernel radius. Hence we link particles that are within one (minimum) kernel length of each other.

This FOF finder yields groupings of star particles and gas elements, from which we compute the baryonic mass $M_{\mathrm{b}}$ and $M_{*}$. Given $M_{\mathrm{b}}$, we then determine $v_{\mathrm{c}}$ for use in our outflow scalings by employing the observed baryonic Tully-Fisher relation (McGaugh et al. 2012) with a redshift evolution as expected from Mo, Mao \& White (1998), namely

$v_{\mathrm{c}}=\left(M_{\mathrm{b}} / 102.329 \mathrm{M}_{\odot}\right)^{0.26178}\left(H(z) / H_{0}\right)^{1 / 3}$,

where $H(z)$ is Hubble's constant at redshift $z$.

\subsection{Feedback from long-lived stars}

Type Ia supernovae (SNIa) and asymptotic giant branch (AGB) stars provide additional energetic feedback. These components provide feedback that is substantially delayed relative to the time of star formation, hence are not represented by our kinetic outflows and must be included separately. Typically, delayed feedback is energetically sub-dominant relative to the processes that drive winds, but they may be important in particular circumstances, hence we include their contributions for completeness.

The SNIa rate is modelled following Scannapieco \& Bildsten (2005) as a prompt component that is concurrent with SNII, and a delayed component that emerges from stars and begins after an age of 0.7 Gyr. The rates are taken from Sullivan et al. (2006), namely $3.9 \times 10^{-4} \mathrm{SNIa}\left(\mathrm{M}_{\odot} / \mathrm{yr}\right)^{-1}$ for the prompt component, and 5.3 $\times 10^{-14} \mathrm{SNIa} \mathrm{M}{ }_{\odot}^{-1}$ for the delayed component (see Oppenheimer \& Davé 2008, for further details).

Each SNIa is assumed to add $10^{51} \mathrm{erg}$ of energy to the surrounding gas. The prompt component energy is added at each timestep to each star-forming gas element, and the delayed component is added to the 16 nearest gas elements to the given star, in a kernel-weighted fashion. To save computation time, we do the delayed feedback for stars only at specific intervals, with the interval scaling roughly inversely with the age of the star, from a minimum of every timestep for ages under $200 \mathrm{Myr}$ to a maximum of every 10 timesteps for stars 2 Gyr and older.

AGB star feedback also adds energy to surrounding gas. We implement the model of Conroy, van Dokkum \& Kravtsov (2015) in which AGB stellar winds are assumed to thermalize with the ambient gas. First, we compute the stellar mass-loss rate assuming a Chabrier (2003) IMF using a lookup table based on Bruzual \& Charlot (2003) models. We then calculate the energy deposition rate based on the mass-loss rate and the velocity difference of the star relative to each neighbouring gas element. We again use the 16 nearest neighbours, in a kernel-weighted fashion. We add the velocity of the stellar wind to this, which we assume to be $100 \mathrm{~km} \mathrm{~s}^{-1}$ (Conroy et al. 2015). In practice, even this large wind stellar velocity that is on the upper end of plausibility makes a negligible difference for most galaxies. This is because if the energy is added to star-forming gas it will quickly radiate away. However, such energy could potentially accumulate into making a substantial impact if the stars are embedded primarily in hot gas, such as in a cluster environment; we will examine this in more detail in future work.

\subsection{Chemical enrichment}

Delayed feedback mechanisms also deposit heavy elements into the surrounding gas. We track the evolution of 11 elements, including hydrogen, helium, and nine metals: $\mathrm{C}, \mathrm{N}, \mathrm{O}, \mathrm{Ne}, \mathrm{Mg}, \mathrm{Si}, \mathrm{S}, \mathrm{Ca}$, and Fe. These elements comprise over 90 per cent of metal mass in the universe.

For SNII yields, we use Nomoto et al. (2006), parametrized as a function of metallicity; we interpolate the yields to the metallicity of the given gas element being enriched. We are using essentially the same yield tables used in Oppenheimer \& Davé (2008), which in Davé et al. (2011b, and other studies) was found to result in metallicities that are too high by a factor of $\sim 2$ compared to the observed mass-metallicity relation. In previous studies (e.g. Davé et al. 2013; Liang et al. 2016) we have simply rescaled metallicities in post-processing, arguing that such changes do not greatly impact the dynamics, and that the predicted yields are uncertain at this level. However, given that cooling and $\mathrm{H}_{2}$-based SFRs depend on metallicity, it is more accurate to reduce the yields during the simulation run. For this reason, we multiply all SNII yields by a factor of 0.5. We add all SNII energy to the star-forming gas element itself, in the instantaneous self-enrichment approximation. On cosmological time-scales, and with sufficient star-forming gas within a given galaxy, this yields a well-converged metallicity distribution.

For SNIa yields, we employ Iwamoto et al. (1999), assuming each SNIa yields $1.4 \mathrm{M}_{\odot}$ of metals. The prompt SNIa component, like SNII enrichment, is done in the instantaneous self-enrichment approximation, while the delayed feedback components are added to the 16 nearest gas element surrounding the given star in a kernelweighted manner.

For AGB stars, we follow Oppenheimer \& Davé (2008) and employ a lookup table as a function of age and metallicity in order to obtain the yields; these yields come from several sources, as described in Oppenheimer \& Davé (2008). We additionally assume 36 per cent helium fraction and a nitrogen yield of 0.00118 . The enrichment, like the energy, is added from stars to the nearest 16 gas elements, kernel-weighted, following the mass-loss rate as computed assuming a Chabrier (2003) IMF.

For the KMT model, we need to provide a total metallicity in solar units, for which we assume a solar abundance taken from Asplund et al. (2009). We do not include metal diffusion or mixing in these models to avoid an extra free parameters, since it is unclear how much metal mixing should be occuring (Schaye, Carswell \& Kim 2007).

\subsection{Quenching feedback}

With only stellar feedback as described above, massive galaxies still form stars too rapidly and the stellar mass function is not truncated at the high-mass end (e.g. Gabor \& Davé 2010). Hence an additional 'maintenance mode' source of energetic feedback is required to fully quench massive galaxies (e.g. Croton et al. 2006; Somerville et al. 2008). Here we follow the model in Gabor \& Davé (2012, 2015), who argued that keeping gas hot in massive haloes is sufficient to yield quenched massive galaxies in agreement with mass function and red sequence observations. 
We quench galaxies by heating all gas in massive haloes except gas that is self-shielded. We compute the self-shielded cold gas fraction for each gas element using the prescription described in Rahmati et al. (2013), which parametrizes the effective strength of the photoionizing background impinging on gas as a function of density and redshift. This ionizing background strength is then used to compute the cold gas fraction (which includes both neutral and molecular gas) using a rate balance equation as described in Popping et al. (2009). Gas is considered self-shielded if its cold gas fraction after applying self-shielding is above 10 per cent. By only heating non-self-shielded gas, most of the neutral and molecular hydrogen within hot haloes is unaffected, ensuring that these cold gaseous components are only directly affected by physical processes such as stripping rather than our quenching prescription.

We apply this quenching heating in massive haloes with a mass that exceeds the quenching mass given by

$M_{\mathrm{q}}=(0.96+0.48 z) \times 10^{12} \mathrm{M}_{\odot}$.

This formula is obtained from the analytic equilibrium model of Mitra, Davé \& Finlator (2015) as the best-fitting parametrization of $M_{\mathrm{q}}$ required to match various observational constraints from $z=0-2$.

Gas in haloes above $M_{\mathrm{q}}$ that is not self-shielded and is below the virial temperature $T_{\text {vir }}$ is heated to 20 per cent above virial temperature $T_{\text {vir }}$ at each timestep. We calculate $T_{\text {vir }}=9.52 \times 10^{7} M_{\mathrm{h}}^{1 / 3} \mathrm{~K}$ (Voit 2005), where we obtain the halo mass $M_{\mathrm{h}}$ using an FOF algorithm with a linking length of 0.16 times the mean interparticle spacing. This FOF algorithm is different than the one used to compute $M_{*}$ and $v_{\mathrm{c}}$, and is only run every 0.5 per cent of a Hubble time only on dark matter particles since it has substantial computational cost; gas and star particles are linked to haloes via their nearest dark matter particle.

We stress that this is a phenomenological model intended to capture the most important effects of whatever microphysics actually drive quenching. Specifically, we are not saying that haloes simply 'self-quench' in the simulations (owing to e.g. virial shocks or simple cooling physics). Quite the opposite: if we only include hydrodynamics, cooling, star formation, and stellar feedback physics, we see far too much star formation at high masses, as noted above. This model directly requires some additional feedback mechanism to be the source of (considerable) additional energy to maintain the virial temperatures of halo gas. The physical mechanisms of this feedback remain uncertain, however - hence the empirical model adopted here. The most likely candidate is feedback from a supermassive black hole, which many groups have shown can plausibly inject sufficient energy far out in the halo and resolve the cooling flow problem (Croton et al. 2006; Somerville et al. 2008; Schaye et al. 2015). In future work, we will explore what the empirical model here requires in terms of energetic input, and how this compares to e.g. the kinetic luminosity function of AGN jets. In these simulations (with $\sim \mathrm{kpc}$ resolution), however, any model for black hole formation/seeding, dynamical evolution, merging, fuelling/growth, and feedback would be necessarily sub-resolution. As such, we would in any case simply adjust the sub-resolution parameters until they produced the same effects as our quenching model here. Our method here thus allows us to more directly isolate what needs to occur on resolved scales in the simulation, in order to match massive galaxy observations.

\subsection{Runs}

All simulations assume a cosmology consistent with Planck Collaboration XIII (2015) 'full likelihood' constraints: $\Omega_{\mathrm{m}}=0.3$,
$\Omega_{\Lambda}=0.7, \Omega_{\mathrm{b}}=0.048, H_{0}=68 \mathrm{~km} \mathrm{~s}^{-1} \mathrm{Mpc}^{-1}, \sigma_{8}=0.82$, and $n_{\mathrm{s}}=0.97$. We generate initial conditions with these parameters using MUSIC (Hahn \& Abel 2011). Each runs begins at $z=249$ in the linear regime.

Our fiducial simulation consists of a $50 \mathrm{~h}^{-1} \mathrm{Mpc}$ randomly selected cubical cosmological volume, with additional simulations used to examine early galaxy evolution having $25 h^{-1} \mathrm{Mpc}$ and $12.5 h^{-1} \mathrm{Mpc}$ box sizes (these are separate runs, not sub-volumes extracted from the larger run). Each run evolves $512^{3}$ gas fluid elements (i.e. mass-conserving cells) and $512^{3}$ dark matter particles. With these choices, the $12.5 \mathrm{~h}^{-1} \mathrm{Mpc}$ volume's halo mass resolution (i.e. 64 dark matter plus 64 gas elements) is $\approx 10^{8} \mathrm{M}_{\odot}$, which means that this run resolves essentially all hydrogen cooling haloes back to the earliest epochs. We will also show results from $25 h^{-1} \mathrm{Mpc}$, $2 \times 256^{3}$ element runs to examine variations in hydrodynamics methodology.

Table 1 lists our main MUfASA run parameters. The $50 h^{-1} \mathrm{Mpc}$ boxes is evolved to a final redshift of $z_{\text {end }}=0$, while the 25 and $12.5 h^{-1} \mathrm{Mpc}$ volumes down to $z_{\text {end }}=2$ owing to both computation expense and the fact that these volumes are too small to be representative at later epochs. We also list the galaxy stellar mass resolution limit $M_{\star, \min }$ in each run, taken to be 32 star particle (or equivalently, gas element) masses. We have found that stellar mass functions are generally well-converged to this limit, but other quantities such as star formation histories can require a higher mass threshold for convergence (e.g. Davé et al. 2011a).

We output 135 snapshots down to $z=0(85$ to $z=2)$ for each run. We analyse the snapshots using $\mathrm{SPHGR}^{4}$ (Thompson 2015), which identifies galaxies using SKID and haloes using ROCKSTAR (Behroozi, Wechsler \& Conroy 2013), and then links them via their positions. SPHGR goes on to calculate many basic properties of the galaxies and haloes such as $M_{*}$ and SFR, and finally outputs a PYTHON 'pickle' file for each snapshot that contains all this information, including member particle lists. All plots in this paper are produced directly from these SPHGR pickle files.

\section{STELLAR MASS FUNCTIONS}

The primary observational benchmark for testing galaxy formation models is the GSMF. The GSMF has now been measured robustly out to $z \sim 3$, covering about 90 per cent of cosmic stellar mass buildup, thanks in particular to improving deep near-infrared surveys such as the Cosmic Assembly Near-IR Deep Extragalactic Legacy Survey (CANDELS; Grogin et al. 2011; Koekemoer et al. 2011) and the FourStar Galaxy Evolution Survey (ZFOURGE; Tomczak et al. 2014). Measurements at higher redshifts are also improving rapidly (e.g. Duncan et al. 2014; Song et al. 2015), though they typically rely on less certain conversions between ultraviolet luminosity and stellar mass. Matching the GSMF and its evolution is thus a key test of whether a model reproduces the bulk of galaxy growth over cosmic time.

It remains a significant challenge for galaxy formation models, be they semi-analytic (SAMs) or hydrodynamic, to match the GSMF to within uncertainties at all redshifts (Somerville \& Davé 2015). SAMs can reproduce the $z=0$ GSMF quite well, typically because they are tuned to do so, while hydrodynamic simulations have recently achieved improved levels of success with $\lesssim \times 2$ discrepancies at $z=0$. Moving to higher redshifts, most models overproduce the GSMF below the knee at $z \sim 1-2$, and some underproduce the

\footnotetext{
${ }^{4}$ http://sphgr.readthedocs.org/en/latest/
} 
GSMF at $z \gtrsim 4$. These differences show up prominently when considering the star formation histories of galaxies, where models characteristically deviate from that inferred for real galaxies particularly at lower masses in the sense of having overly early stellar growth in low-mass galaxies (White, Somerville \& Ferguson 2015). In this section, we will test the MUFASA simulations against observations using the evolution of the GSMF from $z=6 \rightarrow 0$.

\subsection{Comparisons to observations}

Fig. 1 shows the GSMF from our MUFASA simulations at $z=0$, $1,2,3,4,6$. The three box sizes are colour coded as navy blue $\left(12.5 h^{-1} \mathrm{Mpc}\right)$, cyan $\left(25 h^{-1} \mathrm{Mpc}\right)$, and crimson red $\left(50 h^{-1} \mathrm{Mpc}\right)$. Only galaxies with stellar masses above the galaxy mass resolution limit listed in Table 1 are included in each case. We compare to observations (black circles, with Poisson errors) at $z=0$ from the Galaxy and Mass Assembly (GAMA) survey by Baldry et al. (2012) and from Bell et al. (2003), at $z=1,2,3$ to combined CANDELS and ZFOURGE data from Tomczak et al. (2014), and at $z=4$, 6 to CANDELS data from Song et al. (2015). For Tomczak et al. (2014), to obtain the GSMF at $z=1$ we average the quoted values at $z=0.75-1$ and $z=1-1.25$ weighted by the number of galaxies in each bin, and analogously we compute a weighted average of $z=1.5-2$ and $z=2-2.5$ to obtain the $z=2$ points shown; for $z=3$, we quote the $z=2.5-3$ results, which is likely a slight overestimate. While we only choose to compare to one data set so we can more easily engage in quantitative statistical comparisons, various current GSMF observations do not differ significantly at $z \lesssim 2$ (e.g. Somerville \& Davé 2015).

In Table 1 , we quote a reduced $\chi^{2}$ value comparing the simulation data to observational data at each redshift, for each run. To obtain this, we compute error bars on the simulation values as the variance in the GSMF over eight sub-octants of each simulation volume. This approximately accounts for both cosmic variance and Poisson errors, though generally the Poisson errors are sub-dominant. This then produces error bars (asymmetric in log space), which are displayed in Fig. 1. We then compute $\chi^{2}$ from these error bars in log (not linear) space, so that a GSMF that is e.g. low by a factor of 2 will have the same $\chi^{2}$ as one that is high by a factor of 2 . We sum $\chi^{2}$ over the range of overlapping masses between the simulation and the data, using the upper error bar if the data are higher than the simulation, otherwise the lower error bar. To get a reduced $\chi_{v}^{2}$ we divide by the number of observed data points. These $\chi_{v}^{2}$ values provide a quantitative assessment of the level of agreement between MUFASA runs and the observed GSMF.

We note that this value of $\chi^{2}$ does not take into account uncertainties in the observations themselves, but as can be seen in Fig. 1 from the statistical error bars shown on the observed points, these uncertainties are generally sub-dominant to cosmic variance uncertainties. There are additional systematic errors in observed GSMF determinations which can be quite significant particularly at high redshifts, but we also ignore these since these are difficult to quantify accurately (see e.g. Mobasher et al. 2015, for more discussion). We also note that we are comparing SKID-derived masses in simulations to (typically) aperture-based masses from various observational samples, which may introduce a significant bias particularly at high masses $\left(M_{*} \gtrsim 10^{11} \mathrm{M}_{\odot}\right)$ where high-Sérsic profiles create extended wings in the surface brightness distribution (Genel et al. 2014; Kravtsov, Vikhlinin \& Meshscheryakov 2014). Hence the comparisons at the highest masses should be regarded with some additional observational uncertainty, but our values for $\chi_{v}^{2}$ are generally driven by low $M_{*}$ where our cosmic variance er- rors are smaller. Finally, we note that our cosmic variance errors may still be underestimated owing to our smaller volume, as Genel et al. (2014) found somewhat larger uncertainties from $25 h^{-1} \mathrm{Mpc}$ sub-volumes in Illustris. All of these additional aspects would go towards lowering our quoted $\chi_{v}^{2}$ values.

We now examine how well the mass functions agree with observations at various redshifts. At $z=0$, our $50 h^{-1} \mathrm{Mpc}$ fiducial MUFASA run is in remarkably good agreement with the Baldry et al. (2012) GSMF. It faithfully reproduces the observed low-mass slope down to our galaxy mass resolution limit, showing that our star formation feedback algorithm does very well at properly suppressing the low-mass end. At high masses, there is a sharp turnover towards an exponential that has been difficult to achieve in previous models, and highlights the success of translating the evolving quenching mass determined from the equilibrium model of Mitra et al. (2015) into full hydrodynamic simulations. There may be a very slight overproduction of the highest mass galaxies, albeit well within cosmic variance uncertainties, but we note that there is some uncertainty in the masses of massive ellipticals owing to how the galaxy is separated from intracluster light (Kravtsov et al. 2014). As listed in Table 1, the reduced $\chi_{v}^{2}=0.20$ at this redshift relative to Baldry et al. (2012), which quantifies the excellent agreement with observations. The agreement is slightly less good versus Bell et al. (2003), with $\chi_{v}^{2}=0.58$, driven mostly by the reduced high-mass GSMF in this determination.

At $z=1$, the MUFASA mass function is in good agreement with the Tomczak et al. (2014) observations, with $\chi_{v}^{2} \approx 1$. There is a slight excess in the sub- $M^{\star}$ range; this becomes more prominent at $z=2$, which has a comparable $\chi_{v}^{2} \approx 1.1$. The disagreement is driven most strongly by the lowest observed point, which may also be the most uncertain owing to incompleteness in the observations. In contrast, Reddy \& Steidel (2009) determined a steeper faint-end slope of -1.7 from rest-UV observations, in much better agreement with MUFasa. Upcoming observations with the James Webb Space Telescope should definitively settle this issue.

At $z=2$ we see, in both the observations and the $50 h^{-1} \mathrm{Mpc}$ simulation, a turn-down at the highest masses where our quenching model starts to suppress massive galaxy growth. At this redshift, $M_{\mathrm{q}} \approx 10^{13} M_{\odot}$, which means that galaxies with $M_{*} \gtrsim 10^{11} \mathrm{M}_{\odot}$ can begin to be affected. The onset of quenched massive galaxies between $z \sim 2-3$ is in general agreement with observations (e.g. Kriek et al. 2009). Interestingly, the most actively star-forming galaxies in the universe at $z \sim 2.5$ have halo masses of $\sim 10^{13-13.5} \mathrm{M}_{\odot}$ (Wilkinson et al. 2016), which corroborates our value of $M_{\mathrm{q}}$ at these epochs.

Since our smaller volumes are evolved to $z=2$, we can here examine the resolution convergence among all three volumes. Broadly, the three runs lie within cosmic variance uncertainties of each other. However, in detail we see that there is a systematic trend that higher resolution simulations produce slightly higher mass functions in the overlapping mass range. Over a factor of 64 in mass resolution between the 50 and $12.5 h^{-1} \mathrm{Mpc}$ volumes, the mass function at $M_{*} \approx 10^{9-9.5}$ is increased by about a factor of 2 . At higher masses, the GSMF in the smallest volume start to drop low because it cannot form enough massive galaxies. The $25 \mathrm{~h}^{-1} \mathrm{Mpc}$ interpolates this trend, being slightly higher at low $M_{*}$ compared to the fiducial volume, and slightly lower at high $M_{*}$. We note that the $\chi_{v}^{2}$ values for the smaller volumes are still close to unity, so the agreement with data is still satisfactory, although in part this owes to our smaller volumes having larger cosmic variance uncertainties.

At $z=3$, all the volumes' GSMFs are essentially power laws over the modelled mass range. The agreement is excellent with all 

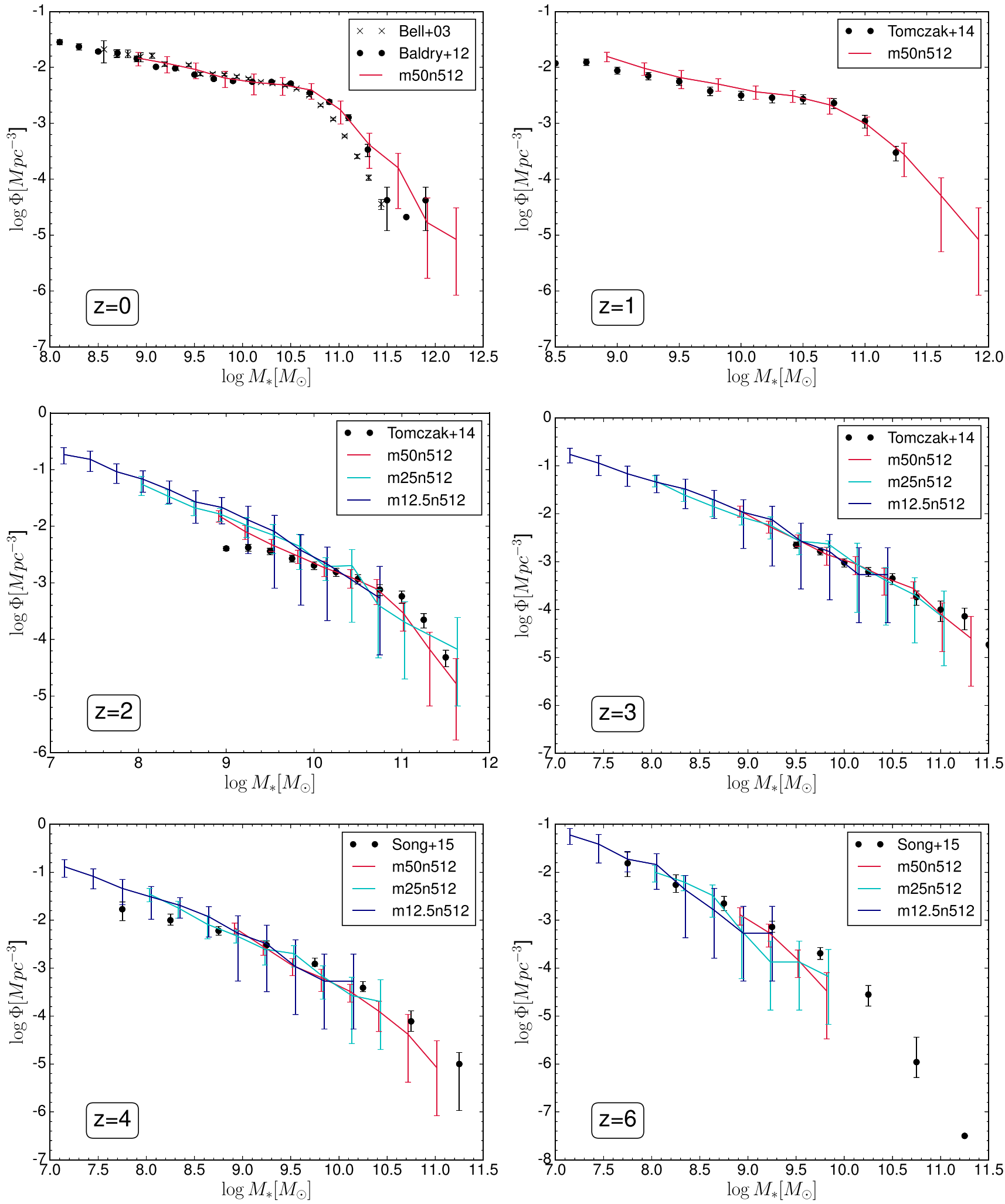

Figure 1. GSMFs at $z=0,1,2,3,4,6$. Dark red, cyan, and dark blue curves show the results from our 50, 25 , and $12.5 h^{-1}$ Mpc volumes, respectively. Simulated galaxies are included down to the stellar mass resolution limits listed in Table 1. Uncertainties are computed from cosmic variance over eight sub-octants of each simulation volume. Observations are shown as solid points from Baldry et al. (2012) at $z \sim 0$, Tomczak et al. (2014) at $z=1-3$, and Song et al. (2015) at $z=4,6$. 
three simulations, with $\chi_{v}^{2} \lesssim 0.3$. However, we note that the data used is actually from galaxies at $z=2.5-3$, so at $z=3$ it is possible that the data should be slightly lower than indicated. Hence as a check we analysed a $z=2.7$ simulation output and compared with this data, and still found very good agreement with $\chi_{v}^{2} \lesssim 0.5$. The resolution convergence among the three volumes here, as well as at all higher redshifts, is excellent.

At higher redshifts $(z=4,6)$, we begin to see slight discrepancies at the high-mass end, in the sense that the MUFASA runs do not produce enough high-mass galaxies. It is already slightly evident at $z=4-$ while the smallest volume has $\chi_{v}^{2}<1$, the larger volumes show $\chi_{v}^{2}=1.3$. The discrepancy becomes somewhat more significant at $z=6$, particularly in the $50 \mathrm{~h}^{-1} \mathrm{Mpc}$ volume, where $\chi_{v}^{2} \approx 2$. The other volumes, probing only lower masses, are not as strongly discrepant, having $\chi_{v}^{2} \leq 1$ at $z=6$. This discrepancy could be the result of the very high bias of early massive galaxies, which reside in large haloes that are not properly represented even in the $50 \mathrm{~h}^{-1} \mathrm{Mpc}$ volume. Alternatively, it could be that the limited resolution does not capture very early star formation in the largest density perturbations. Hence both resolution and volume effects go towards underproducing early stellar mass. We note that another high- $z$ GSMF determination from CANDELS by Duncan et al. (2014) yields generally higher values, which would be further discrepant; the determination from Grazian et al. (2015), on the other hand, are comparable to that of Song et al. (2015). None the less, it is worth noting that are at present substantial systematic uncertainties in deriving stellar masses at these redshifts.

We note that at $z \gtrsim 2$, and particularly at $z \gtrsim 4$, the observational GSMF determinations are subject to significant systematic uncertainties, and various determinations often lie significantly outside each others' formal statistical uncertainties (Ilbert et al. 2013; Muzzin et al. 2013; Tomczak et al. 2014; Mobasher et al. 2015). For this reason, one should use caution in interpreting the agreement or lack therefore with models at these redshifts, as improving observations may shift these values non-trivially. None the less, much of the disagreement between data sets occurs at high $M_{*}$, whereas our $\chi_{v}^{2}$ is driven by our agreement at low $M_{*}$ where our cosmic variance errors are small. For now, we claim that our agreement is comparably good against most recent data sets at these redshifts, but that future observational determinations may yet alter this.

In summary, MUFASA does an excellent job of matching the observed GSMF from $z=4 \rightarrow 0$, in all cases to within $\lesssim 1.2 \sigma$ in simulation cosmic variance. This agreement is unprecedented, and suggest that MUFASA achieves an impressive level of realism in modelling galaxy stellar growth across cosmic time. The agreement at the massive end owes to our evolving halo quenching mass, and suggests that this simple description provides a good match to the truncation of star formation across cosmic time (as argued e.g. in Gabor \& Davé 2012, 2015). It also illustrates the synergy between constraints derived from the analytic equilibrium model and detailed hydrodynamic simulations. At high redshifts, the data are still subject to significant systematic uncertainties, hence we await upcoming deeper observations particularly using the James Webb Space Telescope to more robustly constrain these models. None the less, our high-resolution simulations agree quite well with currently available reionization-epoch GSMF observations, suggesting that simulations that resolve all $\mathrm{H}$-cooling haloes are robust for such studies. Our runs also show reasonable resolution convergence, albeit with a noticeable trend for higher resolution simulations to produce slightly higher GSMFs at the low-mass end.

\subsection{Comparison to other simulations}

Recently, major cosmological galaxy formation simulation projects have set new standards for the number of fluid elements and input physics. Illustris (Genel et al. 2014; Vogelsberger et al. 2014), EAGLE (Crain et al. 2015; Schaye et al. 2015), and MassiveBlack-II (MB-II; Khandai et al. 2015) each model around $1800^{3}$ fluid elements, making them the (computationally) largest cosmological hydrodynamic simulations of galaxy formation evolved to $z=0$ to date. Illustris employs AREPO, EAGLE uses a modern SPH variant, and MB-II uses GADGET-3 with entropy-conserving SPH (Springel 2005). While the dynamic range of these simulations is a remarkable computational achievement, it is still insufficient to directly model the feedback processes that regulate galaxy evolution. Hence like MUFASA, they utilize sub-grid representations for star formation and black hole feedback in order to suppress galaxy growth. The differences in their GSMF predictions are likely driven primarily by variations in feedback recipes, as opposed to variations in hydrodynamic methodology (see e.g. Hopkins 2013, and Section 5.1).

Illustris and MB-II employ decoupled winds, phenomenologically similar to MUFASA. Illustris uses scalings for energy-driven winds, namely $v_{\mathrm{w}} \propto v_{\mathrm{c}}^{-2}$; this is similar to that predicted from high-resolution zooms by Christensen et al. (2016), although the amplitude assumed in Illustris is many times higher. MB-II uses a constant mass loading factor and wind speed similar to the original Springel \& Hernquist (2003b) recipe. Such a 'constant wind' model gives roughly the correct global stellar mass density, but does not fare as well at reproducing GSMFs (e.g. Oppenheimer et al. 2010; Davé et al. 2011a). EAGLE, in contrast, does not decouple winds, but rather assumes that ISM gas particles accumulate SN energy until they can be instantaneously heated to a high temperature, typically $\gg 10^{7} \mathrm{~K}$ where cooling times are long and hence the thermal pressure can drive an outflow. While hydrodynamics remain turned on, cooling is effectively turned off as the particle accumulates SN energy, which occurs for tens of dynamical times, and hence this model is also phenomenological. In this way, the approach has similarities to the blast wave cooling shutoff model of e.g. Governato, Willman \& Mayer (2007) and Stinson et al. (2013). None the less by tuning this prescription, it is possible to nicely reproduce the $z=0$ stellar mass function, with a key aspect being that the given amount of SN energy from star formation is preferentially injected into higher density gas (Crain et al. 2015). While differing in approach, all these simulations have claimed to be in broad agreement with observations of the GSMF.

In Fig. 2, we compare the GSMF of the MUFASA fiducial $50 \mathrm{~h}^{-1} \mathrm{Mpc}$ run to the Illustris simulation (Vogelsberger et al. 2014, orange dashed line;), the EAGLE reference model (McAlpine et al. 2016, dark blue dashed line;), and the MB-II simulation (Khandai et al. 2015, magenta dashed line) at $z=0,1,2$, and 4 . The first two are reproduced from Somerville \& Davé (2015), while the latter data were kindly provided by A. Tenneti and T. DiMatteo. We omit error bars that were shown in Fig. 1 to better see the differences, but we include the same observations as were shown in that figure for reference.

At $z=0$, the MUFASA and EAGLE simulations both do an excellent job of reproducing the observed GSMF, with MUFASA doing somewhat better at capturing the sharp knee of the mass function. Crain et al. (2015) describes the process of tuning EAGLE's input physics in order to obtain this agreement, though it is worth noting that their mass function mostly emerged rather naturally, and much of the tuning was done to match galaxy sizes. In our case, we mostly constrained our input physics using higher resolution simulations 

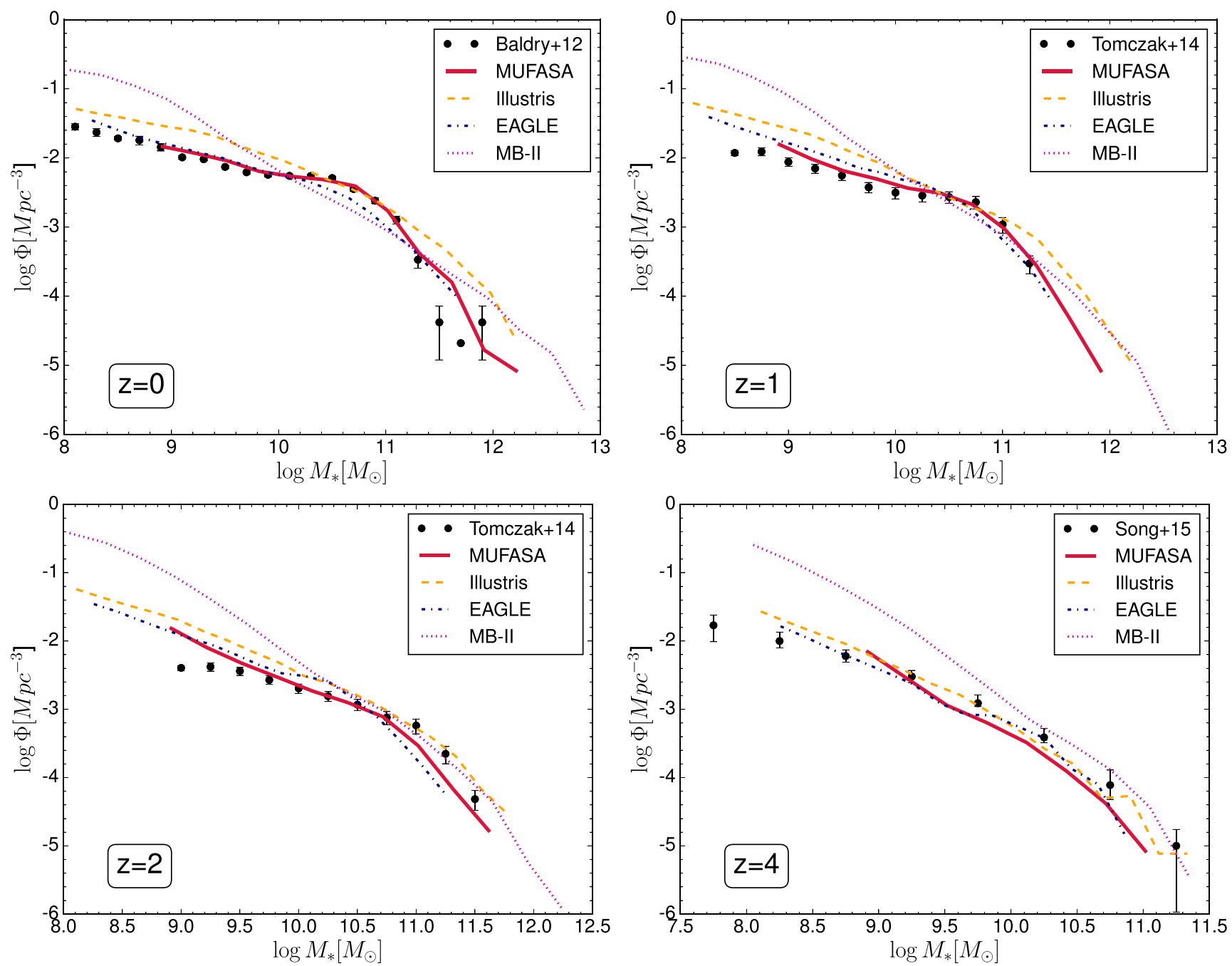

Figure 2. GSMFs at $z=0,1,2,4$ as in Fig. 1, with uncertainties omitted for clarity and the same colour scheme as before, now compared to results from the Illustris (orange dashed; Vogelsberger et al. 2014), EAGLE (dark blue dashed; Schaye et al. 2015), and MassiveBlack-II (magenta dashed; Khandai et al. 2015) simulations. Observations are shown as described in Fig. 1. MUFASA produces better agreement with observations than other current models from $z \sim 0-2$, and slightly worse at $z \sim 4$.

or analytic models, but we still had to mildly tune some parameters such as the outflow velocity. Meanwhile, Illustris clearly overproduces galaxies at both low and high masses, and only agrees well around $M^{\star}$ (i.e. the knee). MB-II shows the characteristic steep mass function that their 'constant wind' feedback model is known to produce, which greatly overpredicts small galaxies and underpredicts the GSMF around $M^{\star}$ (see also Davé et al. 2011a).

At $z=1$, the discrepancies between simulations and data starts to grow, particularly below $M^{\star}$. All the simulations overpredict the low-mass end to varying levels. Illustris and MB-II are quite high, and EAGLE is mildly high compared to observations. MUFASA also overpredicts the low-mass end, but to a lesser extent than any other model, making it clearly the best fit to the intermediate-redshift GSMF.

At $z=2$, the discrepancies seen at $z=1$ for low-mass galaxies persist; generally, the simulations are significantly higher than observations here, with MUFASA providing the closest match. At the massive end, Illustris and MB-II nicely reproduce the turnover, but EAGLE cuts off the GSMF at too low a mass. MUFASA also truncates the GSMF slightly early, albeit less so than EAGLE. Part of this may owe to the smaller $50 h^{-1} \mathrm{Mpc}$ box size of MUFASA, relative to the other simulations which have $\sim 70-100 h^{-1}$ Mpc box sizes.

At $z=4$, Illustris and EAGLE do somewhat better than MUFASA, as our fiducial MUFASA run produces too low a GSMF. Our $25 h^{-1} \mathrm{Mpc}$ volume which has comparable resolution to those runs, as well as our $12.5 h^{-1} \mathrm{Mpc}$ run, show better agreement as seen in Fig. 1. As mentioned previously, the deficit in the $50 \mathrm{~h}^{-1} \mathrm{Mpc}$ run may owe to the lack of resolution to start galaxy growth early enough, combined with the lack of volume required to include growth of the largest haloes. None the less, MUFASA is still within $\sim 1 \sigma$ of cosmic variance, so it is possible that the chosen initial conditions unluckily do not produce enough early massive galaxies.

While the MUFASA runs presented here lack a dynamic range comparable to these larger simulations, they do offer several modelling advantages. Compared to MB-II, MUFASA employs a hydro solver that has been shown to yield better results for key test problems (Hopkins 2015), and additionally our outflow scalings better reproduce the observed GSMFs at the low-mass end. Compared 


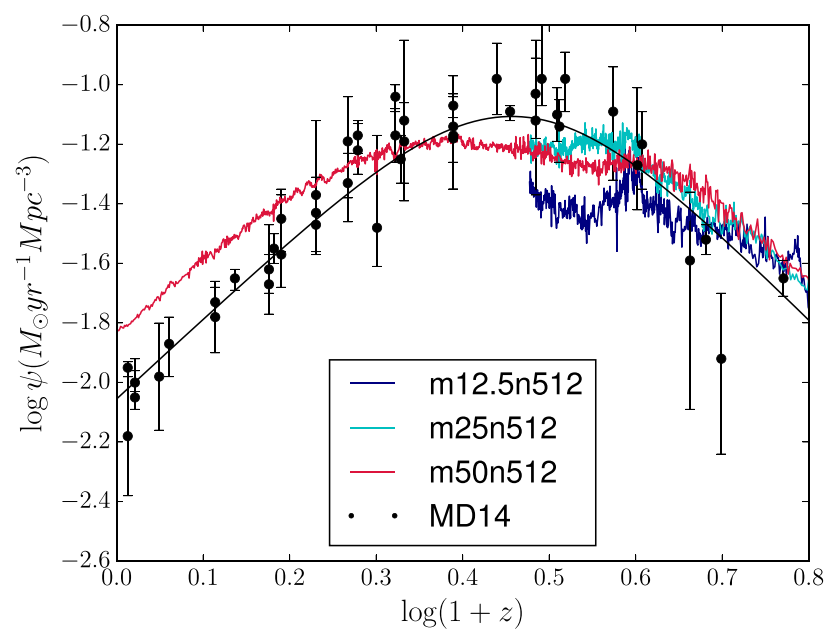

Figure 3. Cosmic SFRD evolution as a function of $\log (1+z)$, in our 50 (red), 25 (cyan), and $12.5 h^{-1} \mathrm{Mpc}$ (dark blue) MUFASA simulations. A fit to observations from Madau \& Dickinson (2014) is shown as the black line in each panel, and the individual data points from their compilation are also represented.

to Illustris, MUFASA reproduces observed GSMFs significantly more closely over most of cosmic time. Generally, EAGLE and MUFASA yield comparably good mass functions, with MUFASA slightly better from Cosmic Noon until today, and EAGLE slightly better at $z=4$. MUFASA has some disadvantages as well. Besides less dynamic range, we also do not yet explicitly track central black hole growth and feedback as the other simulations do (this is in progress; see e.g. Anglés-Alcázar et al. 2016). Nevertheless, compared to recent state-of-the-art galaxy formation simulations, MUFASA generally is as good or better at reproducing the observed stellar mass buildup in galaxies across the majority of cosmic time as traced by the GSMF.

\section{GALAXY GROWTH RATES}

\subsection{Global SFR density}

The growth of galaxy stellar mass is reflected in the SFRs of galaxies across cosmic time. A key barometer of this is the evolution of the cosmic star formation rate density (SFRD), commonly known as a Lilly-Madau plot. We note that the cosmic SFRD is not an ideal test of models, because it is an integrated quantity that can be sensitive to observational integration limits, and individual simulations only cover a limited range of the total cosmic star formation. Hence discrepancies compared to observations may reflect limitations of volume or resolution rather than intrinsic model failings. None the less, the SFRD evolution is a often-used benchmark and highlights some important points about the characteristics of galaxy growth.

Fig. 3 shows the cosmic SFRD $(\psi)$ evolution as a function of $\log (1+z)$. Our three volumes $\left(50,25,12.5 h^{-1} \mathrm{Mpc}\right)$ are shown as the crimson, cyan, and navy blue lines, respectively. Here, we have plotted the SFR directly output during the simulation run as the stellar mass formed per unit time, which displays small-time-scale fluctuations owing to the stochastic nature of our star formation algorithm; this employs no selection criterion, and even includes star formation in unresolved galaxies. For comparison, data from a recent compilation by Madau \& Dickinson (2014) are shown in black, including a line for their global best-fitting relation along with the individual compiled data points; these have been divided by 1.7 in order to correct from their assumed Salpeter IMF to a Chabrier IMF.

The fiducial $50 h^{-1} \mathrm{Mpc}$ simulation qualitatively follows the observed trend faithfully: there is a rapid rise of $\psi$ from $z \sim 8 \rightarrow 3$, then it is relatively constant until $z \sim 1.5$, and then it falls rapidly towards the present day. The evolution since $z \sim 1.5$ generally follows the power-law expectation from cosmological accretion, as has been noted by many previous works (e.g. Somerville \& Davé 2015). In detail, MUFASA underpredicts the global SFR during Cosmic Noon (which we will discuss in Section 4.4), and overpredicts the SFR at lower redshifts $(z \lesssim 1.5)$ by about 50 per cent. This is particularly curious in light of the good agreement with the evolution of the GSMF. It is also opposite to the discrepancy versus the recent models such as Illustris, EAGLE, and the SAM of Henriques et al. (2015), which tend to underpredict the SFRD at low- $z$ while still generally agreeing with GSMF evolution. The SFRD peak at $z \sim 1-3$ is also broader in MUFASA than in the data, which likewise mimics the trend in other current models. It is not obvious how one reconciles all these models and observations; in part it may reflect systematic uncertainties in SFR measures, or suggest that a more careful comparison should be done to account for observational selection effects. We will break this down versus $M_{*}$ in Section 4.4 to gain more insights into the differences.

The $25 h^{-1}$ Mpc run matches the $50 h^{-1}$ Mpc quite well over the redshift range probed. This is a bit of a coincidence, as we have seen in the GSMF that the low-mass end is slight overproduced in this run, which appears to compensate for the lack of high-mass starforming galaxies owing to the smaller volume. For the $12.5 h^{-1} \mathrm{Mpc}$ run, this coincidental balance fails, as the lack of high-mass starforming galaxies dominates over the mild excess production at low masses. As a result, $\psi$ begins to lie significantly below that of the larger volumes at $z \lesssim 4$. There is also significant stochasticity over long time-scales at $z \lesssim 3$ (in addition to the short stochasticity) because the global star formation is dominated by a handful of the most massive galaxies whose SFRs can vary substantially. Overall, the small-volume results are seen to be not cosmologically representative at $z \lesssim 4$ in terms of large galaxy growth, but the evolution of dwarf galaxies should still be robust. At $z \gtrsim 4$, all our volumes' SFRD values agree with each other, and are within the uncertainties of the Madau \& Dickinson (2014) data.

In summary, the SFRD evolution from $z=6 \rightarrow 0$ is broadly reproduced in our MUFASA runs. This is not surprising given the fact that the GSMF matches reasonably well at all redshifts $z \lesssim 6$. The deviations at $z \lesssim 4$ in the $12.5 h^{-1} \mathrm{Mpc}$ volume highlight incompleteness at the massive end owing to its small volume; deviations at lower redshifts will be discussed Section 4.4.

\subsection{Star formation downsizing}

A key characteristic of the galaxy population is known as 'downsizing', first coined by Cowie et al. (1996), in which star formation shifts from more massive galaxies to lower mass galaxies over time. This results in more massive galaxies having older stellar populations, which has been dubbed 'archaeological downsizing' (Thomas et al. 2005). While downsizing has been referred to as antihierarchical, the qualitative trend is a natural outcome of hierarchical structure formation, since the largest galaxies arise in the largest density perturbations which are able to collapse and start forming stars first (Neistein, van den Bosch \& Dekel 2006). None the less, 


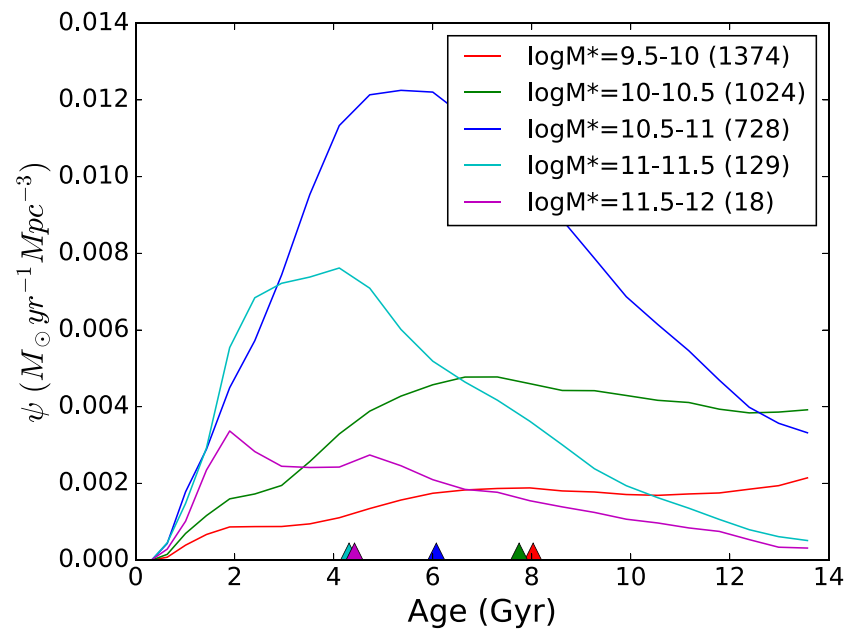

Figure 4. Cosmic SFRD evolution as a function of time in our fiducial $50 h^{-1} \mathrm{Mpc}$ MUFASA run split into bins of final $(z=0)$ galaxy stellar mass as indicated, with the number of $z=0$ galaxies shown in parentheses. Colourcoded upward arrows along the bottom of plot indicate the time at which half the stars have formed within those galaxies. Downsizing is evident, as more massive galaxies have an earlier median star formation time.

quantitatively reproducing the observed growth rates of galaxies as a function of mass has proved challenging for models.

Since the fiducial MUFASA simulation well reproduces the observed stellar mass function covering over 90 per cent of cosmic star formation, it provides a quantitative picture of downsizing as a function of galaxy mass. Fig. 4 illustrates this. Here, we show the star formation history of galaxies sub-divided into five bins of $z=0$ stellar mass, from $10^{9.5}$ to $10^{12} \mathrm{M}_{\odot}$. The time at which half the present-day stellar mass was formed is indicated by the colourcoded upward arrows along the bottom of the plot, for each mass bin. Note that this is computed by examining the formation times of the remaining stellar mass at $z=0$, which is different than the SFR in those galaxies at any particular epoch, owing to the effects of stellar mass-loss; in general, the median SFR will be shifted to slightly earlier times. This also makes no distinction between stellar mass that was formed in the main progenitor versus accreted satellite galaxies. In this sense, our depiction here is most closely related to archaeological downsizing.

Downsizing is clearly evident here - higher mass galaxies have earlier formation epochs. Galaxies with $M_{*}>10^{10.5} \mathrm{M}_{\odot}$, which are predominantly quenched today, have median stellar formation times around $4 \mathrm{Gyr}$, or close to $z \sim 2$. Galaxies with $M_{*} \lesssim 10^{10.5} \mathrm{M}_{\odot}$ in contrast have formation times of $\sim 8 \mathrm{Gyr}$, i.e. $z \sim 0.6$. Galaxies around the knee of the mass function today dominate the global stellar mass, and have a median formation time of around $6 \mathrm{Gyr}$, or $z \sim 1$. Hence our simulations naturally predict that more massive galaxies will host older stellar populations.

The smallest galaxies have a global SFR that steadily increases with time all the way to the present day, which is qualitatively different than galaxies that end up around the knee of the GSMF. Such star formation histories in dwarfs are qualitatively what is required to solve the so-called dwarf galaxy conundrum, in which current models generically predict dwarf galaxy star formation histories that peak at too early epochs (White et al. 2015). We will examine this so-called dwarf galaxy conundrum using MUFASA in more detail in future work.

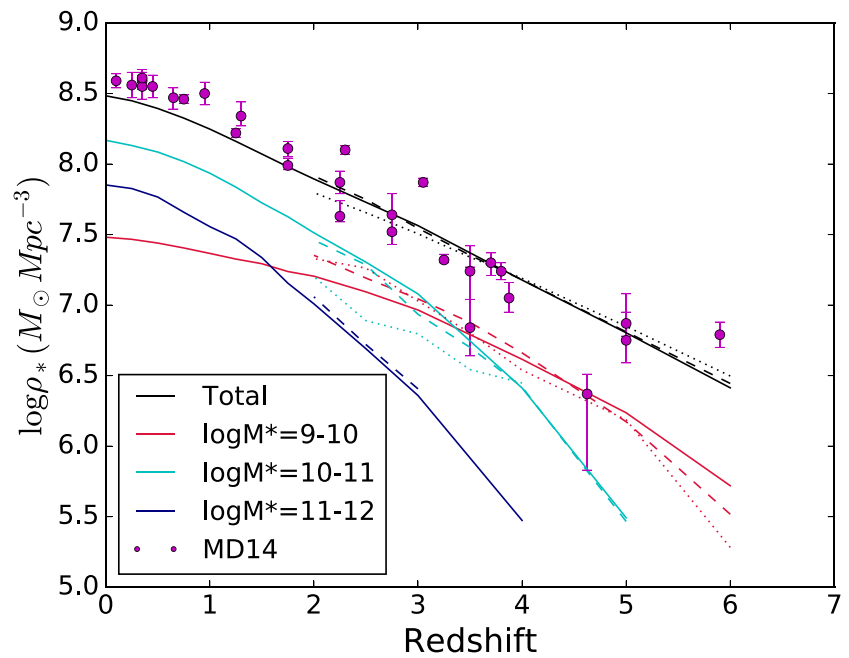

Figure 5. Cosmic stellar mass density evolution as a function of time in our MUFASA runs (black lines). Solid lines show our $50 h^{-1} \mathrm{Mpc}$ run down to $z=0$, while the dashed and dotted lines show the 25 and $12.5 h^{-1} \mathrm{Mpc}$ volumes, respectively. Observational data are shown as compiled by Madau \& Dickinson (2014, various sources described in text). We further sub-divide the global stellar mass into bins of stellar mass (at that epoch) as indicated. The overall stellar mass density is in good agreement with data, and for most of cosmic time is dominated by galaxies with $M_{*}=10^{10-11} \mathrm{M}_{\odot}$.

\subsection{Stellar mass density evolution}

A complementary view of galaxy growth is provided by the evolution of the cosmic stellar mass density. In principle, this should be equivalent to an integral of the Lilly-Madau plot, accounting for stellar evolution. Some discrepancies between these two approaches have been claimed in the past (e.g. Davé 2008; Wilkins, Trentham $\&$ Hopkins 2008), but these have been contradicted by more recent data (Reddy et al. 2012; Madau \& Dickinson 2014); the issue remains unsettled.

Fig. 5 shows the evolution of the cosmic stellar mass density in our three simulations; solid, dashed, and dotted lines correspond to our 50, 25, and $12.5 h^{-1} \mathrm{Mpc}$ runs, respectively. We also show the breakdown in three ranges of stellar mass, the dwarf $\left(10^{9-10} \mathrm{M}_{\odot}\right)$, $M^{\star}\left(10^{10-11} \mathrm{M}_{\odot}\right)$, and massive $\left(10^{11-12} \mathrm{M}_{\odot}\right)$ galaxy regimes, colour coded as indicated in the legend. Finally, the cyan points show some recent (post-2010) data from the compilation of observations by Madau \& Dickinson (2014), namely Moustakas et al. (2013) at low redshifts, Ilbert et al. (2013), Muzzin et al. (2013), and Reddy et al. (2012) out to $z \sim 3$, and Gonzalez et al. (2011), Lee et al. (2012), and Caputi et al. (2011) to higher redshifts.

The total stellar mass density evolution is generally in good agreement with observations. At $z \lesssim 1$ the $50 h^{-1} \mathrm{Mpc}$ box falls slightly below the data, potentially owing to observed galaxies extending to masses below the resolution limit of this simulation. The discrepancy is never more than 30 per cent, none the less it is interesting that this contrasts with the predictions for the Lilly-Madau plot, which show an overprediction of the observed SFRD at these redshifts. This hints at some mismatch between the observed global growth of stars versus the observed global SFRs. At high redshifts the agreement is very good, although the data show more scatter there.

Breaking $\rho_{*}(z)$ down by mass, at early epochs the total mass density is dominated by relatively small galaxies, since larger galaxies have not yet formed. By $z \sim 3-4$, galaxies above $10^{10} \mathrm{M}_{\odot}$ begin to dominate the mass density, which continues all the way until the 

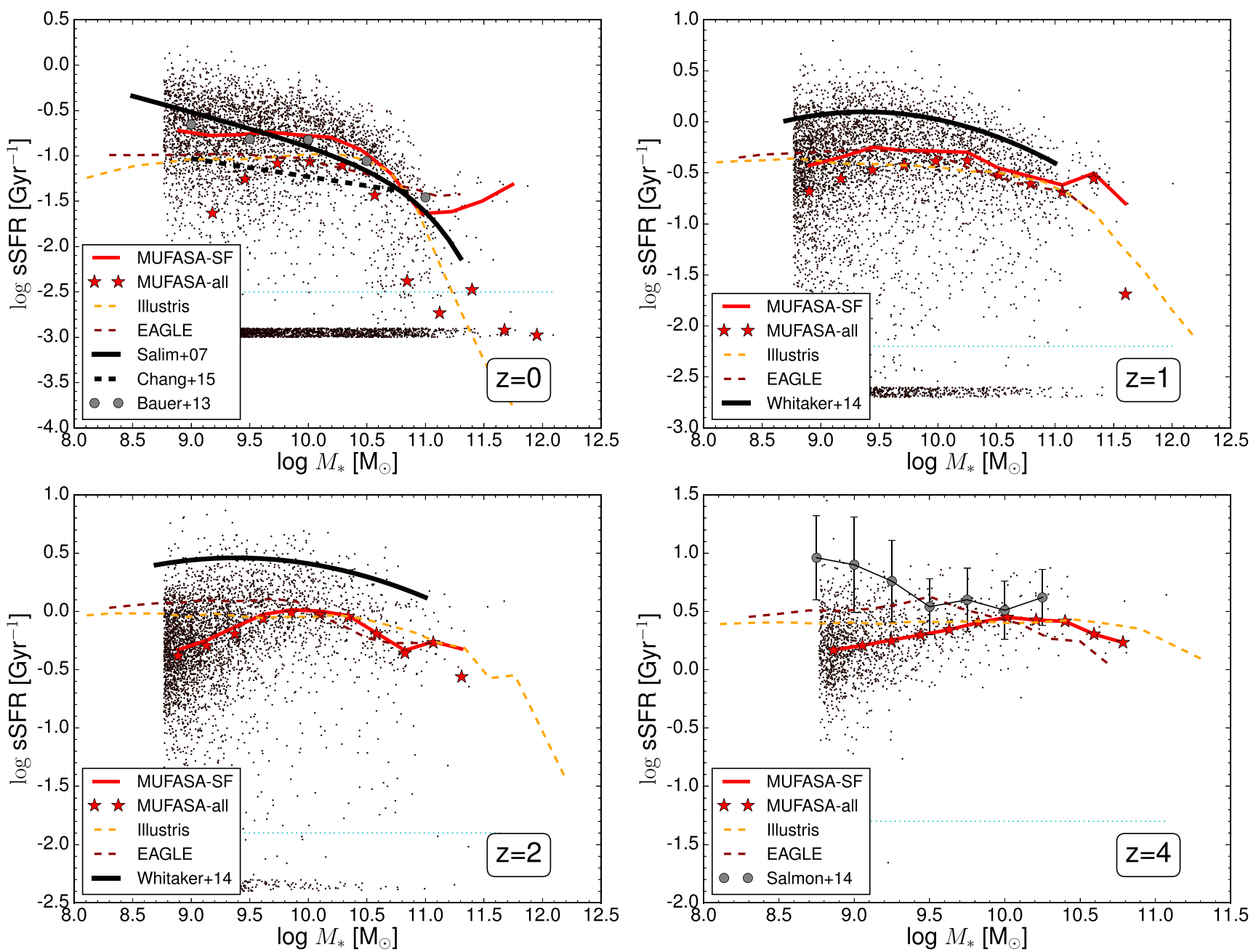

Figure 6. sSFR versus stellar mass for galaxies at $z=0,1,2,4$ in our fiducial $50 h^{-1}$ Mpc MUFASA simulation. Points show individual galaxies, with galaxies having very low sSFR plotted across the bottom (with artificial scatter added for visibility). Thick red lines show a running mean value for all star-forming galaxies, i.e. galaxies above the horizontal dotted cyan line near the bottom. The stars show a running median sSFR, including non-star-forming galaxies. Observations are shown as follows: at $z=0$, the Schechter function fit from Salim et al. (2007, black solid), the fit from Chang et al. (2015, black dashed), and data from Bauer et al. (2013, grey points); at $z=1,2$ we show the polynomial fit to data from Whitaker et al. (2014); while at $z=4$ we show data from Salmon et al. (2015). Finally, the dashed orange and dark red lines show results from Illustris and EAGLE, respectively. The predicted sSFR is in good agreement at $z=0,4$, but is systematically low at $z=1,2$, following the trend seen in previous galaxy formation models.

present day. Larger galaxies do not dominate at early times since few have formed, and do not dominate at late times since their growth is suppressed by quenching. Examining the various box sizes by comparing lines of the same colour, we see that there is generally good convergence even when broken down by mass, though there are variations of up to about 50 per cent.

Overall, MUFASA simulations do a good job of reproducing the cosmic stellar mass density evolution, and the variations between the different resolution boxes is not large. Together with the good match to the Lilly-Madau plot, this demonstrates that MUFASA provides a viable suite of models with which to examine global stellar mass growth.

\subsection{Specific star formation rates}

A key test of how galaxies grow is the evolution of the SFR-stellar mass relation, often called the star-forming galaxy 'main sequence' (Noeske et al. 2007). Now measured out to $z \sim 6$ and beyond, its evolution has proved to be challenging for models to reproduce particularly during Cosmic Noon $(z \sim 1-3)$, with both SAMs and simulations typically predicting specific star formation rates (sSFRs) at $z \sim 2$ that are too low by $\sim \times 2-3$ (Daddi et al. 2007; Davé 2008; Somerville \& Davé 2015; Sparre et al. 2015). Many of these same models, however, also tend to overpredict the GSMF during Cosmic Noon by roughly the same factor, so it has been conjectured that a model that matches the GSMF at $z \sim 1-3$ (and keeps the SFRs the same) would also match the main sequence. Since the MUFASA simulations provide a reasonable fit to the GSMF at $z=1-3$, it is interesting to examine whether they likewise matches the main sequence at these epochs.

Fig. 6 shows sSFR versus $M_{*}$ at $z=0,1,2,4$ in our fiducial $50 h^{-1}$ Mpc MUFASA simulation. Along the bottom are depicted galaxies with very low (typically zero) sSFR, with some artificial spread to enhance visibility. The red solid line shows the running median SSFR as a function of $M_{*}$, for star-forming galaxies defined as those above the cyan dashed line lying just above the quenched 
galaxies. The stars indicate a binned median for all galaxies, including the quenched ones. Observations are shown in black: At $z=0$, we show a Schechter-form fit from Salim et al. (2007, solid black) based data from the Sloan Digital Sky Survey (SDSS) and the Galaxy Evolution Explorer (GALEX), as well as $\mathrm{H} \alpha$-based SFR determinations from the GAMA survey by Bauer et al. (2013, grey points). We also show a fit to the recent SDSS+Wide Infrared Survey Explorer (WISE) determination by Chang et al. (2015, dashed black). At $z=1,2$ we show data from 3D-HST with Spitzer $24 \mu \mathrm{m}$ information by Whitaker et al. (2014), and at $z=4$ from CANDELS by Salmon et al. (2015). Finally, for comparison we show results from the Illustris (orange dashed) and EAGLE (dark red dashed) simulations.

At $z=0$, MUFASA star-forming galaxies show a flat low-mass end and a turn-down at $M_{*} \gtrsim 10^{10.5} \mathrm{M}_{\odot}$ as the general population starts to be quenched (but is still regarded as star-forming by our cut). The predictions are in very good agreement with recent $\mathrm{H} \alpha$ based SFR's from GAMA (Bauer et al. 2013, grey circles), and in reasonable agreement with the SDSS $+G A L E X$ data from Salim et al. (2007), However, they are higher than the sSFR's inferred from full 0.4-22 $\mu \mathrm{m}$ SED fitting including WISE data (Chang et al. 2015). It is beyond the scope of this paper to assess the various differences in observations, but we note that even the present-day SFRs are somewhat controversial.

For comparison, we show results from Illustris (orange; $z=0$ ) and EAGLE (dark red; $z=0.1$ ) simulations, which like MUFASA show a flat $\operatorname{sSFR}\left(M_{*}\right)$ at low masses (see also Davé et al. 2011a), but with an amplitude that is lower by $\sim \times 1.5-2$. These are generally in better agreement with the Chang et al. (2015) data. Hence even simulations that nicely match the GSMF such as MUFASA and EAGLE can have substantially different $z=0$ sSFR values for starforming galaxies. As mentioned earlier, the global SFRD in these other simulations tend to be lower than that in MUFASA, and from the $\operatorname{sSFR}\left(M_{*}\right)$ comparison this is likewise seen to be the case for individual galaxies with $M_{*} \lesssim 10^{10.7} \mathrm{M}_{\odot}$. There are also a handful of massive MUFASA galaxies that are still star-forming as well, which contribute non-trivially to the global SFRD. We will examine the massive galaxy population and its colours in an upcoming paper.

The stars show the binned median values from MUFASA now including the quenched galaxies. Such galaxies dominate by number at low and high masses - the median values (stars) at $M_{*} \sim 10^{9}$ and $M_{*} \gtrsim 10^{11} \mathrm{M}_{\odot}$ are actually in the quenched population. At low masses these are typically stripped satellites, while at high masses they are quenched centrals. Observations generally do not suggest quite such a dominant population of low-mass quenched satellites, but such satellites are also difficult to observe, so selection effects may be important. We leave a more thorough investigation of central versus satellite populations for future work.

Moving to $z=1$, the MUFASA predictions clearly lie below the data, typically by a factor of up to 2 relative to the Whitaker et al. (2014) data across the resolved mass range. Illustris and EAGLE are comparable. Indeed, such a discrepancy is generically seen among models of all types (Somerville \& Davé 2015). The number of quenched galaxies is markedly less, so that the medians are now generally within the star-forming population.

At $z=2$, the agreement gets even worse - the deficit of MUFASA versus the Whitaker et al. (2014) data is now $\times 3$ across most of the mass range. This is comparable to or even lower than the predictions from Illustris (Sparre et al. 2015) and EAGLE (Schaye et al. 2015). There are very few quenched galaxies by this epoch, so one cannot easily appeal to burstiness to explain the discrepancy - besides the fact that the observed tight main-sequence scatter limits the contribution of starbursts (Rodighiero, Daddi \& Baronchelli 2011).

This discrepancy at $z \sim 2$ remains a major puzzle, and it starkly contrast with the fact that MUFASA nicely reproduces the evolution of the GSMF around this redshift range. If the Universe is producing stars at a rate $\sim 3$ times higher than MUFASA at $z \sim 2$, and the GSMF agrees at $z \sim 2$, then where do all those stars end up? They do not appear to produce an excess in the observed GSMF relative to MUFASA at $z \sim 1$; if anything, MUFASA lies slightly above the observed GSMF at $z=1$. Hence it seems that the rate of stellar growth as tracked by the GSMF is not straightforward to reconcile with that measured directly via SFRs. It could be that the observed SFRs are simply too high owing to calibration issues, but the uncertainties in UV $+24 \mu \mathrm{m}$ SFRs are generally thought to be significantly smaller than the discrepancy. As the conjecture in Bastian, Covey \& Meyer (2010) states, any problem in galaxy formation can be resolved with a suitable choice of IMF, and in this case a top-heavy or bottom-light IMF remains a viable if unpopular solution (Davé 2008; Wilkins et al. 2008).

The discrepancy could also be solved by raising $M_{*}$ values at $z \sim 2$ by $\sim \times 3$ via some unforeseen systematic errors. Given that current GSMF data at this epoch is now based on deep near-IR data that probes well beyond the $4000 \AA$ break, it would be quite surprising if the stellar mass estimates were so far off; current methods for estimating $M_{*}$ typically do not differ systematically by such large amounts (Mobasher et al. 2015). Furthermore, this would shift the GSMF to quite high values, and would result in their being more massive galaxies at $z=2$ than today. Hence this solution seems untenable.

Finally, at $z=4$ the dynamic range spanned by observations is relatively small, but the disagreements versus the data are clearly less, particularly for larger star-forming galaxies. MUFASA shows little trend with mass at this redshift, and perhaps even has a positive slope at $M_{*} \lesssim 10^{10} \mathrm{M}_{\odot}$. The Salmon et al. (2015) data plotted show a rise towards the lowest masses that at face value is divergent from the model predictions, but incompleteness in identifying low-SFR galaxies via Lyman break selection at these redshifts may mitigate this disagreement. The discrepancy in $\operatorname{sSFR}\left(M_{*}\right)$ versus models thus seems to be primarily restricted to Cosmic Noon $(z \sim 1-3)$, as has long been noted (e.g. Davé 2008).

In summary, MUFASA shows similar trends to previous models in matching observations at low and high redshifts, but falling low throughout Cosmic Noon. At $z=0$ there are substantial differences versus recent simulations at the low-mass end, with MUFASA showing higher levels of present-day star formation in star-forming dwarfs, although quenched dwarfs dominate by number. At intermediate redshifts, MUFASA mimics the shape if not amplitude of $\operatorname{sSFR}\left(M_{*}\right)$, with a positive $\operatorname{sSFR}\left(M_{*}\right)$ slope at low masses and a negative one at high masses. The high observed sSFR values across all masses at $z \sim 1-3$ remain a persistent quandary for modern galaxy formation models, even ones that match the observed GSMF evolution through that epoch.

\section{CODE VARIATIONS}

A major advantage of GIZMO is that it has the ability to trivially switch between four hydrodynamic solvers while holding all other input physics fixed. Hence it provides an ideal platform for testing the sensitivity of results such as the GSMF to hydrodynamic methodology. To contextualize the differences resulting from numerical methodology, we explore how key input physics choices change the GSMF. 

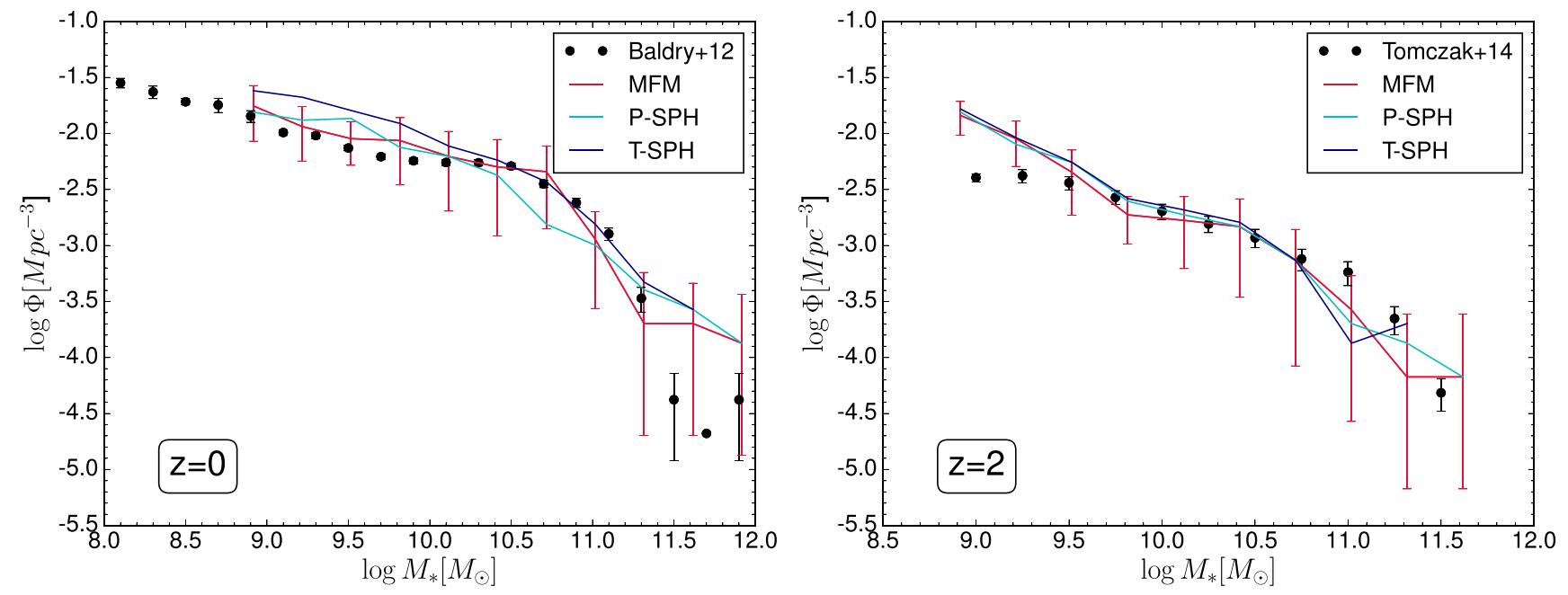

Figure 7. GSMFs at $z=0,2$ in $25 h^{-1} \mathrm{Mpc}, 2 \times 256^{3}$ particle simulations run with three hydrodynamic solvers within GIZMO: our fiducial MFM (crimson), modern P-SPH (cyan), and T-SPH (navy). All are started with identical initial conditions, with identical feedback physics. Cosmic variance error bars are only shown for the MFM case; the others are comparable. MFM and T-SPH tend to produce larger galaxies than P-SPH, more prominently so at later epochs, while MFM produces a flatter low-mass slope.

\subsection{Sensitivity to hydrodynamics methodology}

GIZMO contains four hydrodynamics solvers: MFM, which is our fiducial choice; meshless finite volume (MFV), which is a massadvecting moving mesh-like scheme; P-SPH (Hopkins 2013), which is a pressure-energy formulation of SPH that improves surface instability handling; and T-SPH, which is a density-energy formulation of SPH. These are described in more detail in Hopkins (2015, Appendix F). We note that T-SPH employs some new features in GIZMO relative to older SPH codes, such as an improved artificial viscosity and artificial conduction; see Hopkins (2015) for details. Since MFV does not strictly conserve mass within fluid elements (though it does so approximately), it is not obvious how reliably it will function with the kinetic outflows, stellar mass-loss, etc. hence we will not consider this scheme here. Instead, we will focus on comparing the mass-conserving schemes MFM, P-SPH, and T-SPH.

Fig. 7 shows the GSMF at $z=0$ (left) and $z=2$ (right) in runs with MFM, P-SPH, and T-SPH. These runs have a box size of $25 h^{-1} \mathrm{Mpc}$ and $2 \times 256^{3}$ particles, but in every other way are identical to our fiducial $50 \mathrm{~h}^{-1} \mathrm{Mpc}$ MUFASA run, excepting the hydro solver which is only identical for the MFM case. Observations are shown as described in Fig. 1 for reference, although the focus here is on comparing among the hydro solvers rather than comparing to data. Error bars are again computed as cosmic variance over eight sub-octants within each simulation volume (only shown for the MFM case; the others are similar but omitted for clarity), and they are larger than in the full $50 \mathrm{~h}^{-1} \mathrm{Mpc}$ MUFASA run owing to the smaller volume.

We first note that the $25 h^{-1}$ Mpc MFM run produces a GSMF that is statistically identical to the $50 \mathrm{~h}^{-1} \mathrm{Mpc}$ run. This is not surprising, as they have identical numerical resolution and input physics, but it is reassuring that these simulations display good GSMF convergence with volume, even though there are many fewer galaxies in the smaller volume.

Comparing amongst the various hydro solvers, at early epochs $(z=2)$ the GSMFs are very similar. In other words, kinetic outflows are suppressing early galaxy formation in a manner that is essentially independent of the hydro solver. Indeed, the differences at $z \geq 3$ (not shown) are even smaller.

By $z=0$, the three hydro solvers diverge non-trivially in their predictions. As discussed in Oppenheimer et al. (2010), the contribution from wind recycling (i.e. the re-accretion of previously ejected material) to stellar mass growth starts to be significant at $z \lesssim 2$, and becomes dominant by $z=0$. Our preliminarily interpretation is thus that the differences between hydro solvers owe primarily to differences in the way that galactic outflows interact with the surrounding medium and rejoin the accretion flow. This is consistent with the idea that the main improvement in MFM over SPH is handling two-phase instabilities and shocks, which are important mechanisms for how winds interact with circumgalactic gas.

T-SPH tends to produce about 50 per cent more galaxies at any given $M_{*}$ compared to P-SPH. Meanwhile, both SPH codes produce a steeper GSMF than MFM. In Oppenheimer et al. (2010), it was noted that the flattening of the GSMF in the sub- $M^{\star}$ regime is achieved in simulations by wind recycling. If this is likewise the case in these simulations (as we expect), then we can infer that winds recycle more effectively in MFM than in SPH.

The detailed reasons for these variations are not entirely obvious. Our preliminary interpretation for difference between P-SPH and $\mathrm{T}$-SPH is as follows. In flows with large variations of internal energy $u$ from particle-to-particle, P-SPH, because it kernel-averages the pressure over all neighbours' $u$ as opposed to just using the particle's $u$ times density, a single very high- $u$ particle can raise the pressure of all its neighbours. This means that all of them together can do more PdV work than a single hot particle could. While this is done in an energy-conserving way, it can effectively enhance the ability of lone, hot particles to add substantial pressure. In our case, the thermal heating of the winds results in such lone hot particles, which then increases the pressure in CGM gas upon recoupling, more so in P-SPH than T-SPH. This can then expand the CGM gas and lower the amount of wind re-accretion, which results in a lower GSMF for 

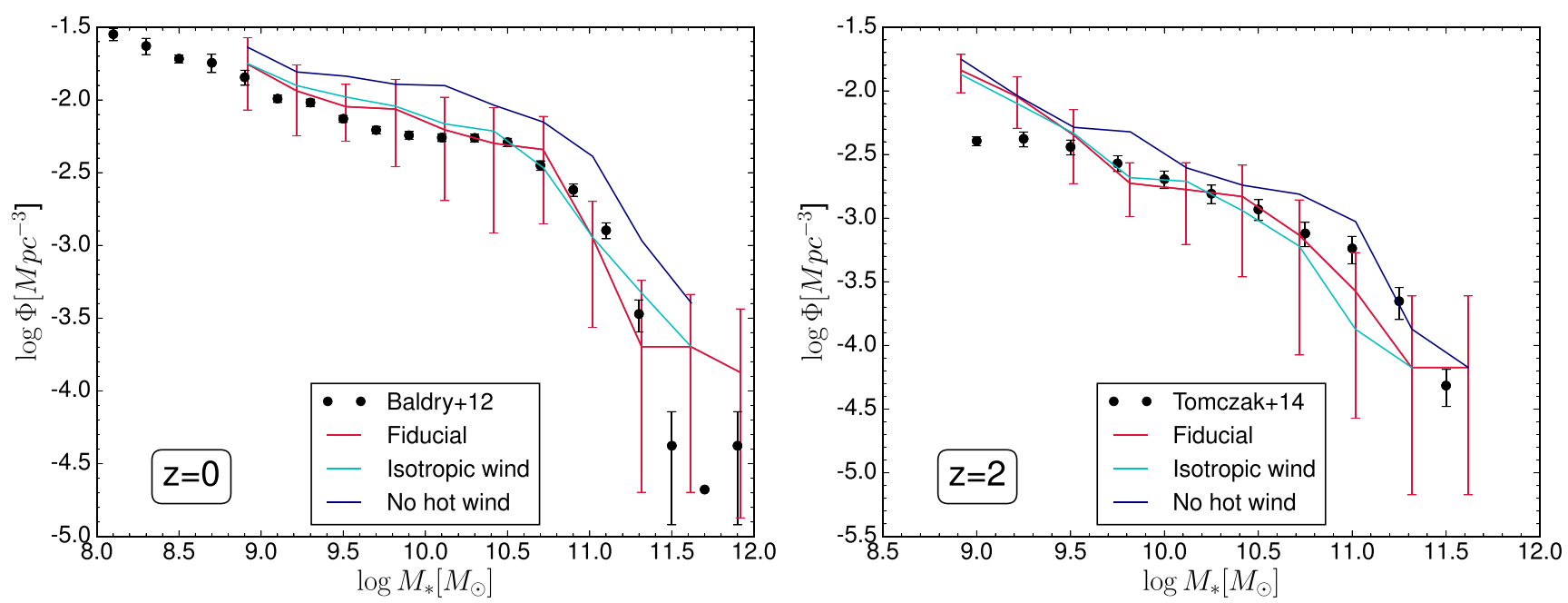

Figure 8. GSMFs at $z=0,2$ in $25 h^{-1} \mathrm{Mpc}, 2 \times 256^{3}$ particle simulations runs comparing our fiducial feedback prescription (crimson) with one with purely cool winds (navy) and having isotropic wind ejection (cyan). Cosmic variance error bars are only shown for the fiducial case; the others are comparable. The inclusion of 30 per cent hot winds in the fiducial case significantly suppresses galaxy growth, more so at later epochs, while ejecting winds isotropically does not make a substantial difference.

P-SPH. While we have not confirmed this effect in detail, we believe this is a plausible interpretation for the origin of the discrepancy between P-SPH and T-SPH. Meanwhile, MFM generally increases the amount of mixing, which increases the amount of recycling relative $\mathrm{SPH}$, and hence flattens the GSMF.

This highlights a key point that, although we have left all the feedback physics and numerical parameters fixed in the code between the three methods, there are inherent differences between the methods that can interact with feedback in ways that depend on methodology. For instance, Hopkins (2015) points out that the effective resolution in discontinuities and shocks is higher in MFM versus SPH owing to less smoothing, which could play a role in more accurately modelling the hot wind interactions. We also note that the details of SPH scheme can matter, and differences can arise when injecting energy using SPH owing to the manner in which energy or entropy is smoothed among neighbours (Schaller et al. 2015). The sensitivity to methodology presented here therefore should be regarded as specific to both the hydro scheme and the feedback implementation, and not a generic result. None the less, it gives some idea as to the level of variations that can occur among modern methods.

It is of course not immediately evident which hydro solver yields a more correct answer. None the less, in idealized test cases, MFM handles discontinuities and shear flows in a more realistic manner than SPH (Hopkins 2015), which is consistent with results in more realistic circumstances (Wetzel et al. 2016). We expect that issues of hydrodynamics will be more important in massive galaxies that contain hot gaseous haloes, where two-phase discontinuities are stronger; hence our $25 \mathrm{~h}^{-1} \mathrm{Mpc}$ volume is perhaps not ideal for these tests. For now, we preliminarily conclude that there are differences of up to $\sim \times 2$ for $z \lesssim 1$ GSMF predictions based purely on which hydrodynamics solver is used, while at higher $z$ the predictions are less sensitive to this.

\subsection{Sensitivity to outflow parametrizations}

MUFASA includes new implementations of feedback prescriptions, so it is interesting to investigate how some of these novel aspects impact the predicted GSMFs. Here we examine two aspects, namely two-phase winds and the wind ejection direction, and determine how large of an impact they make on the resulting GSMFs.

Fig. 8 shows the GSMF at $z=0$ (left) and $z=2$ (right) in simulations where we turn off two-phase winds and eject all winds cool (i.e. at $T \sim 10^{4} \mathrm{~K}$ ), shown in navy blue, while the cyan curve shows the result of ejecting winds isotropically rather than outwards in the $\boldsymbol{v} \times \boldsymbol{a}$ direction. It is clear that the choice of wind direction has a very minimal impact on the resulting GSMF, though we might expect that it could have signatures in the enrichment of the CGM.

In contrast, turning off the hot wind component has a large impact, somewhat more so at later epochs; at $z \gtrsim 4$ (not shown) the differences are fairly small. Evidently, the hot component of outflows adds significant preventive feedback, thereby suppressing galaxy growth. Number densities are lowered by $\sim \times 2-3$, with a somewhat greater impact for more massive galaxies. Ejecting some fraction of winds hot is therefore an important aspect of how MUFASA obtains good agreement with the GSMF.

Besides indirectly impacting the GSMF, two-phase outflows are also likely to significantly impact the thermal state of the CGM. Hence observations of quasar absorption lines around galaxies such as the COS-Haloes project (Tumlinson et al. 2013) can in principle provide constraints on outflow temperatures. Previous simulations that ejected winds purely cold tended to underpredict the amount of absorption from ions tracing warmer gas such as $\mathrm{O}$ vI (Ford et al. 2016). In future work we will examine whether the new two-phase outflow model produces better agreement.

In summary, the thermal state of the outflows adds an important component of preventive feedback that helps suppress galaxy formation in accord with observations. The choice of wind direction, in contrast, has minimal impact. Zoom simulations that selfconsistently drive outflows via SN heating can in principle directly predict the temperature distribution of outflowing gas, and we hope that future such efforts will aim to quantify predictions for the thermal state of outflowing gas, in addition to the mass-loss rate and velocity. We note that the differences in the GSMF owing to 
two-phase winds exceed the differences owing to choice of hydrodynamics methodology discussed in the last section; this is consistent with previous investigations (e.g. Schaye, Dalla Vecchia \& Booth 2010; Davé et al. 2011a; Hopkins et al. 2014).

\section{SUMMARY}

We present a new set of cosmological hydrodynamic simulations called MUFASA run using the meshless GODUNOV finite mass hydrodynamics method in the new GIZMO code (Hopkins 2015). We include updated recipes for star formation and feedback, including molecular hydrogen based star formation following KMT, two-phase kinetic outflows with scalings based on the FIRE zoom simulations of Muratov et al. (2015), and halo mass based quenching with an evolving quenching mass scale taken from analytic equilibrium model constraints by Mitra et al. (2015). Our feedback prescriptions are still phenomenological, but the parameters are mostly taken from high-resolution simulations or independently constrained analytic models rather than tuned by directly matching our simulations to observations. We run simulations with 50,25 , and $12.5 h^{-1} \mathrm{Mpc}$, the large fiducial volume to $z=0$, and the latter two to $z=2$ to study the high-redshift population in more detail, each having 2 $\times 512^{3}$ particles and sub-kpc resolution. We test our models against measures of galaxy growth from $z=6 \rightarrow 0$, particularly the GSMF, the global evolution of stellar mass density and SFR, and the stellar mass-SFR relation. We briefly investigate how much choices of hydrodynamics methodology and outflow modelling impact the predicted GSMF.

Our main results are summarized as follows.

(i) The fiducial $50 h^{-1} \mathrm{Mpc}$ MUFASA simulation agrees very well with the observed evolution of the GSMF from $z=6 \rightarrow 0$, usually matching data to $\sim 1 \sigma$ in cosmic variance uncertainty or better, and providing unprecedented agreement to this key barometer for galaxy formation models. The resolution convergence between the three volumes at $z \geq 2$ is reasonable good, with a systematic increase of $\times 2$ in galaxy number density over a factor of 64 in mass resolution.

(ii) Compared to recent hydrodynamic simulations Illustris, EAGLE, and MB-II, MUFASA reproduces the observed GSMF about equally well or better at any given redshift. Particularly, it has been challenging for models to match the GSMF at $z \sim 1-3$, which MUFASA does quite well. This suggests that the cosmic growth rate of stellar mass in galaxies can now be viably modelled by cosmological hydrodynamic simulations.

(iii) The cosmic SFRD evolution is in general agreement with observations, albeit too low during Cosmic Noon and too high at $z \lesssim 1.5$. The cosmic stellar mass density growth shows a strong trend of archaeological downsizing across the full range of $M_{*} \sim$ $10^{9.5-12} \mathrm{M}_{\odot}$, namely that more massive galaxies contain stars that on average formed earlier. The most massive galaxies have a median stellar age of $\approx 9-10$ Gyr $(z \sim 2)$, while small dwarfs show a more protracted, constant or increasing star formation history with a median stellar age of $\approx 5-6$ Gyr $(z \sim 0.6)$.

(iv) The predicted SFR-stellar mass relation (main sequence) is in general agreement with recent $\mathrm{H} \alpha$ and UV-based SFR data at $z \approx 0$. But as with previous models, MUFASA has difficulty matching observations at $z \sim 1-3$, falling short in sSFRs at $z=2$ by $\sim \times 3$. It remains a perplexing mystery how the overall GSMF evolution can be so well reproduced, while a direct measure of growth rates in individual galaxies suggests a significant discrepancy through the peak epoch of galaxy growth. (v) Comparing GSMFs between identical runs using MFM, P$\mathrm{SPH}$, and T-SPH shows that hydrodynamics methodology plays a small but non-negligible role in determining the GSMF. It is increasingly influential at late times and in more massive galaxies, presumably because this is where the interactions between outflows and accreting gas become more important for galaxy growth. None the less, such variations are sub-dominant compared to variations in the GSMF owing to choices in feedback. For instance, our twophase winds including a 30 per cent $\mathrm{SN}$-heated component adds substantial preventive feedback that suppresses the GSMF by $\sim \times 2$ 3 by today.

The MUFASA simulations represent a continued shift in the approach towards incorporating sub-grid physics into cosmologicalscale simulations of galaxy formation. MUFASA relies increasingly on the results from very high-resolution zoom simulations and wellconstrained analytic models to constrain sub-grid prescriptions, rather than simple but highly tuned parameterizations. In this way it takes advantage of the true multiscale nature of simulations today, marrying models spanning far greater dynamic range than can be represented in any single simulation. MUFASA's success at reproducing key galaxy observables over most of cosmic time demonstrates that this is a fruitful approach.

Despite being substantially less fine tuned than many previous galaxy formation simulations, we still have free parameters in our outflow modelling that could be more tightly constrained from external models, such as the outflow velocity and thermal state. Furthermore, our current prescription for quenching massive galaxies remains purely heuristic, so must be transitioned to a more physically based model that includes the self-consistent growth of and energy release from central black holes. As our ability to model the physics driving feedback owing to star formation and active galactic nuclei grows, the goal is to replace ad hoc sub-grid prescriptions with direct characterisations from more physically based models. The MUFASA simulations presented here offer an important step in this direction, while providing a state-of-the-art platform to investigate the physical processes driving galaxy evolution across a wide range of mass scales and cosmic epochs.

\section{ACKNOWLEDGEMENTS}

The authors thank D. Anglés-Alcázar, F. Durier, S. Huang, N. Katz, T. Naab, B. Oppenheimer, M. Rafieferantsoa, and J. Schaye for helpful conversations. The authors thank Paul Torrey for providing us with the Illustris data, Rob Crain and Joop Schaye for the EAGLE data, and Ananth Tenneti and Tiziani DiMatteo for the MB-II data. RD and RJT acknowledge support from the South African Research Chairs Initiative and the South African National Research Foundation. Support for RD was also provided by NASA ATP grant NNX12AH86G to the University of Arizona. Support for RJT was provided in part by the Gordon and Betty Moore Foundation's Data-Driven Discovery Initiative through Grant GBMF4561 to Matthew Turk, and by the National Science Foundation under grant \#ACI-1535651. Support for PFH was provided by an Alfred P. Sloan Research Fellowship, NASA ATP Grant NNX14AH35G, and NSF Collaborative Research Grant \#1411920 and CAREER grant \#1455342. The simulations were run on the Pumbaa astrophysics computing cluster hosted at the University of the Western Cape, which was generously funded by UWC's Office of the Deputy Vice Chancellor. These MUFASA simulations were run with revision e77f814 of GIZMo hosted at https://bitbucket.org/rthompson/gizmo. 


\section{REFERENCES}

Agertz O. et al., 2007, MNRAS, 380, 963

Anglés-Alcázar D., Özel F., Davé R., Katz N., Kollmeier J. A., Oppenheimer B. D., 2015, 800, 127

Anglés-Alcázar D., Davé R., Faucher-Giguere C. A., Özel F., Hopkins P. F., 2016, MNRAS, preprint (arXiv:1603.08007)

Asplund M., Grevesse N., Sauval A. J., Scott P., 2009, ARA\&A, 47, 481

Baldry I. K. et al., 2012, MNRAS, 421, 621

Bastian N., Covey K. R., Meyer M. R., 2010, ARA\&A, 48, 339

Bauer A. E. et al., 2013, MNRAS, 434, 209

Behroozi P. S., Wechsler R. H., Conroy C., 2013, ApJ, 770, 57

Bell E. F., McIntosh D. H., Katz N., Weinberg M. D., 2003, ApJS, 149, 289

Bruzual G., Charlot S., 2003, MNRAS, 344, 1000

Caputi K. I., Cirasuolo M., Dunlop J. S., McLure R. J., Farrah D., Almaini O., 2011, MNRAS, 413, 162

Chabrier G., 2003, PASP, 115, 763

Chang Y.-Y., van der Wel A., da Cunha E., Rix H.-W., 2015, ApJS, 219, 8

Christensen C., Davé R., Governato F., Pontzen A., Brooks A., Munshi F., Quinn T., Wadsley J., 2016, ApJ, 824, 57

Conroy C., van Dokkum P. G., Kravtsov A., 2015, ApJ 803, 77

Cowie L. L., Songaila A., Hu E. M., Cohen J. G., 1996, AJ, 112, 839

Crain R. A., 2015, MNRAS, 450, 1937

Croton D. J. et al., 2006, MNRAS, 365, 11

Cullen L., Dehnen W., 2010, MNRAS, 408, 669

Daddi E. et al., 2007, ApJ, 670, 156

Davé R., 2008, MNRAS, 385, 147

Davé R., Oppenheimer B. D., Finlator K. M., 2011a, MNRAS, 415, 11

Davé R., Finlator K. M., Oppenheimer B. D., 2011b, MNRAS, 416, 1354

Davé R., Katz N., Oppenheimer B. D., Kollmeier J. A., Weinberg D. H., 2013, MNRAS, 434, 2645

Dehnen W., Aly H., 2012, MNRAS, 425, 1068

Duncan K., 2014, MNRAS, 444, 2960

Durier F., Dalla Vecchia C., 2012, MNRAS, 419, 465

Faucher-Giguere C. A., Lidz A., Zaldarriaga M., Hernquist L., 2009, ApJ, 703,1416

Faucher-Giguere C. A., Kereš D., Dijkstra M., Hernquist L., Zaldarriaga M., 2010, ApJ, 725, 633

Ferland G. J., 2004, BAAS, 36, 1574

Ford A. B. et al., 2016, MNRAS, 459, 1745

Gabor J. M., Davé R., 2010, MNRAS, 407, 749

Gabor J. M., Davé R., 2012, MNRAS, 427, 1816

Gabor J. M., Davé R., 2015, MNRAS, 447, 374

Gabor J. M., Davé R., Oppenheimer B. D., Finlator K. M., 2011, MNRAS, 417, 2676

Genel S. et al., 2014, MNRAS, 445, 175

González V., Labbé I., Bouwens R. J., Illingworth G., Franx M., Kriek M., 2011, ApJ, 735, L34

Governato F., Willman B., Mayer L., 2007, MNRAS, 374, 1479

Grazian A. et al., 2015, A\&A, 575, 96

Grogin N. A. et al., 2011, ApJS, 197, 35

Haardt F., Madau P., 2012, ApJ, 746, 125

Hahn O., Abel T., 2011, MNRAS, 415, 2101

Henriques B. M. B., White S. D. M., Thomas P. A., Angulo R., Guo Q., Lemson G., Springel V., Overzier R., 2015, MNRAS, 451, 2633

Hernquist L., Katz N., 1989, ApJS, 70, 419

Hopkins P. F., 2013, MNRAS, 428, 2840

Hopkins P. F., 2015, MNRAS, 450, 53

Hopkins P. F., Raives M. J., 2016, MNRAS, 455, 51

Hopkins P. F., Kereš D., Oñorbe J., Faucher-Giguere C. A., Quataert E., Murray N., Bullock J. S., 2014, MNRAS, 445, 581

Ilbert O. et al., 2013, A\&A, 556, 55

Iwamoto K., Brachwitz F., Nomoto K., Kishimoto N., Umeda H., Hix W. R., Thielemann F.-K., 1999, ApJS, 125, 439

Kennicutt R. C., 1998, ApJ, 498, 541

Kereš D., Katz N., Weinberg D. H., Davé R., 2005, MNRAS, 363, 2

Khandai N., Di Matteo T., Croft R., Wilkins S., Feng Y., Tucker E., DeGraf C., Liu M.-S., 2015, MNRAS, 450, 1349
Kim J.-H. et al., 2014, ApJS, 210, 14

Koekemoer A. M. et al., 2011, ApJS, 197, 36

Kollmeier J. A. et al., 2014, ApJ, 789, L32

Kravtsov A., Vikhlinin A., Meshscheryakov A., 2014, ApJ, preprint (arXiv:1401.7329)

Kriek M., van Dokkum P. G., Franx M., Illingworth G. D., Magee D. K., 2009, ApJ, 705, 71

Krumholz M. R., McKee C. F., Tumlinson J. T., 2009, ApJ, 693, 216 (KMT)

Krumholz M. R., McKee C. F., Tumlinson J. T., 2011, ApJ, 729, 36

Łokas E. L., Mamon G. A., 2001, MNRAS, 321, 155

Lee K.-S. et al., 2012, ApJ, 752, 66

Liang L., Durier F., Babul A., Davé R., Oppenheimer B. D., Katz N., Fardal M., Quinn T., 2016, MNRAS, 456, 4266

McAlpine S. et al., 2016, Astron. Comput., 15, 72

McGaugh S. S., 2012, AJ, 143, 40

Madau P., Dickinson M., 2014, ARA\&A, 52, 415

Martin C. L., 2005, ApJ, 621, 227

Mitra S., Davé R., Finlator K., 2015, MNRAS, 452, 1184

Mo H. J., Mao S., White S. D. M., 1998, MNRAS, 295, 319

Mobasher B., 2015, ApJ, 808, 101

Monaghan J. J., 1992, ARA\&A, 30, 543

Moustakas J. et al., 2013, ApJ, 767, 50

Muratov A. L., Kereš D., Faucher-Giguere C. A., Hopkins P. F., Quataert E., Murray N., 2015, MNRAS, 454, 2691

Muzzin A. et al., 2013, ApJ, 777, 18

Navarro J., Frenk C. S., White S. D. M., 1997, ApJ, 490, 493

Neistein E., van den Bosch F. C., Dekel A., 2006, MNRAS, 372, 933

Noeske K. G. et al., 2007, ApJ, 660, L43

Nomoto K., Tominaga N., Umeda H., Kobayashi C., Maeda K., 2006, Nucl. Phys. A, 777, 424

Oppenheimer B. D., Davé R., 2006, MNRAS, 373, 1265

Oppenheimer B. D., Davé R., 2008, MNRAS, 387, 577

Oppenheimer B. D., Davé R., Kereš D., Katz N., Kollmeier J. A., Weinberg D. H., 2010, MNRAS, 406, 2325

Planck Collaboration XIII, 2015, preprint (arXiv:1502.01589)

Popping A., Davé R., Braun R., Oppenheimer B. D., 2009, A\&A, 504, 15

Rahmati A., Pawlik A. H., Raičević M., Schaye J., 2013, MNRAS, 430, 2427

Read J. I., Hayfield T., 2012, MNRAS, 422, 3037

Reddy N. A., Steidel C. C., 2009, ApJ, 692, 778

Reddy N. A. et al., 2012. ApJ 744, 154

Rodighiero G., Daddi E., Baronchelli I., 2011, ApJ, 739, L40

Salim S. et al., 2007, ApJS, 207, 173, 267

Salmon B. et al., 2015, ApJ, 799, 183

Scannapieco E., Bildsten L., 2005 ApJ, 629, L85

Schaller M., Dalla Vecchia C., Schaye J., Bower R. G., Theuns T., Crain R. A., Furlong M., McCarthy I. G., 2015, MNRAS, 454, 2277

Schaye J., Dalla Vecchia C., 2008, MNRAS, 383, 1210

Schaye J., Carswell R. F., Kim T.-S., 2007, MNRAS, 379, 1169

Schaye J., Dalla Vecchia C., Booth C. M., 2010, MNRAS, 402, 1536

Schaye J. et al., 2015, MNRAS, 446, 521

Schmidt M., 1959, ApJ, 129, 243

Sijacki D., Springel V., Di Matteo T., Hernquist L., 2007, MNRAS, 380, 877

Somerville R. S., Davé R., 2015, ARA\&A, 53, 51

Somerville R. S., Hopkins P. F., Cox T. J., Robertson B. E., Hernquist L., 2008, MNRAS, 391, 481

Song M. et al., 2015, ApJ, 791, 3

Sparre M. et al., 2015, MNRAS, 447, 3548

Springel V., 2005, MNRAS, 364, 1105

Springel V., 2010, MNRAS, 401, 791

Springel V., Hernquist L., 2002, MNRAS, 333, 649

Springel V., Hernquist L., 2003a, MNRAS, 339, 289

Springel V., Hernquist L., 2003b, MNRAS, 339, 312

Steidel C. C., Erb D. K., Shapley A. E., Pettini M., Reddy N., Bogosavljević M., Rudie G. C., Rakic O., 2010, ApJ, 717, 298

Stinson G. S., Brook C., Maccio A. V., Wadsley J., Quinn T. R., Couchman H. M. P., 2013, MNRAS, 428, 129 
Sullivan M. et al., 2006, ApJ, 648, 868

The Enzo Collaboration, 2014, ApJS, 211, 19

Thomas D., Maraston C., Bender R., Mendes de Oliveira C., 2005, ApJ, 621,673

Thompson R., 2015, Astrophysics Source Code Library, 1502, 12

Thompson R., Nagamine K., Jaacks J., Choi J.-H., 2014, ApJ, 780, 145

Thompson R., Davé R., Huang S., Katz N., 2016, MNRAS, preprint (arXiv:1508.01851)

Tomczak A. R., 2014, ApJ, 783, 85

Tumlinson J. et al., 2013, ApJ, 777, 59

Vogelsberger M. et al., 2014, MNRAS, 444, 1518
Voit G. M., 2005, Adv. Space Res., 36, 701

Wetzel A. R., Hopkins P. F., Kim J.-h., Faucher-Giguere C.-A., Keres D., Quataert E., 2016, ApJ, 827, L23

Whitaker K. E. et al., 2014, ApJ, 795, 104

White C. E., Somerville R. S., Ferguson H. C., 2015, ApJ, 799, 201

Wilkins S. M., Trentham N., Hopkins A. M., 2008, MNRAS, 385, 687

Wilkinson A. et al., 2016, MNRAS, preprint (arXiv:1604.00018)

This paper has been typeset from a $\mathrm{T}_{\mathrm{E}} \mathrm{X} / \mathrm{LAT}_{\mathrm{E}} \mathrm{X}$ file prepared by the author. 